hep-th/0012104

RUPT-????

October 26, 2018

\title{
Instabilities in heterotic M-theory induced by open membrane instantons.
}

\author{
Gregory Moore ${ }^{\dagger}$, Grigor Peradze ${ }^{\dagger \diamond}$, and Natalia Saulina ${ }^{\dagger \dagger}$ \\ $\dagger$ Department of Physics, \\ $\diamond$ Department of Physics, \\ Rutgers University \\ Yale University \\ Piscataway, NJ 08854, USA \\ New Haven,CT 06520,USA \\ gmoore@physics.rutgers.edu \\ peradze@physics.rutgers.edu \\ †† Department of Physics, Jadwin Hall \\ Princeton University \\ NJ 08544, USA \\ saulina@princeton.edu
}

\begin{abstract}
We study the effective low energy supergravity of the strongly coupled heterotic string compactified on a Calabi-Yau 3 -fold with generic $E_{8} \times E_{8}$ gauge bundle. We focus on the effective potential for the chiral scalars. The effective superpotential can be studied using the dual 11-dimensional M-theory background involving insertions of M5 branes along an interval. In such backgrounds, in some regions of moduli space, the leading nonperturbative contributions are due to open membrane instantons. These instantons lead to both attractive and repulsive forces between the 5-branes and the orientifold "M9-branes," depending on the region of moduli space. The resulting dynamics on moduli space include a strong coupling dual to the Dine-Seiberg instability, in which the interval grows. We discuss conditions under which the 5-branes are attracted to the wall and comment on the relevance of these results to the study of chirality-changing phase transitions in heterotic M-theory.
\end{abstract}




\section{Contents}

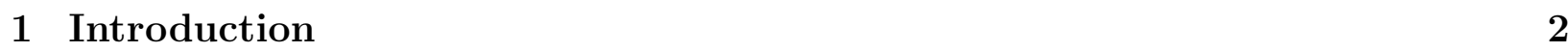

2 Review of heterotic M-theory background with M5-branes on the interval 7

$\begin{array}{lll}3 & \text { M2-brane instantons in } \mathcal{X} \times S^{1} / Z_{2} & 12\end{array}$

4 Calculation of membrane-instanton-induced superpotentials $\quad 15$

4.1 Summary of the computation of $\Delta W \ldots \ldots \ldots$

4.2 Computation of the vertex operator . . . . . . . . . . . . . . . . . . . . . . . 17

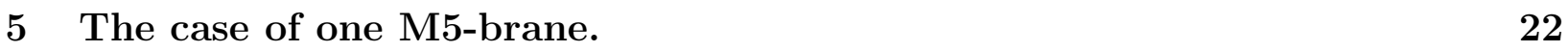

5.1 Superpotential . . . . . . . . . . . . . . . . . . . . 22

5.2 Kahler potential for bulk moduli and charged scalars . . . . . . . . . . . . . 24

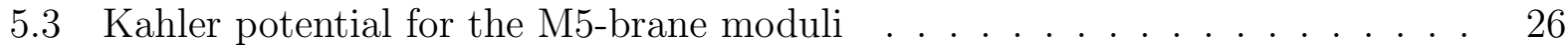

5.4 Potential in the case $h^{(1,1)}=1$. . . . . . . . . . . . . . . . . . . . . . . . . 28

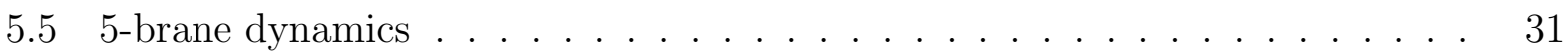

5.6 Conflicting instabilities . . . . . . . . . . . . . . . . . . . 33

6 Multiple covers and chirality changing transitions 34

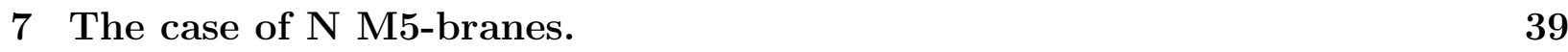

8 Possible future directions and applications $\quad 41$

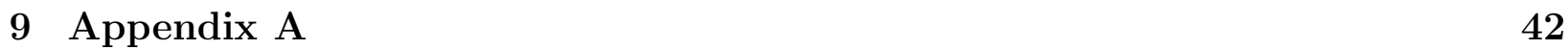

\begin{tabular}{|l|l}
\hline 10 Appendix B & 44
\end{tabular} 


\section{Introduction}

In the past few years there have been significant advances in the study of strongly coupled heterotic string theory, thanks to the formulation in terms of $M$-theory on an interval $S^{1} / Z_{2}$ [1], 2]. In particular, the compactification of $M$-theory on a product of an interval with a Calabi-Yau 3-fold (denoted hereafter by $\mathcal{X}$ ) leads to qualitatively different physics from that of the weakly coupled heterotic string, as first noted in [3, 4, 15].

In heterotic string compactification one must choose an instanton configuration for gauge fields along $\mathcal{X}$. The so-called "standard embedding" identifies the gauge field with the spin connection of the metric. Other choices of gauge instantons, the so-called "nonstandard embeddings," are closely related, in the strongly coupled regime, to backgrounds obtained by including insertions of M5-branes wrapping a product of 4-dimensional spacetime with a holomorphic curve $\Sigma$ in $\mathcal{X}$. At low energies, the physics of such backgrounds is summarized by a complicated $d=4, N=1$ supergravity theory. It has been shown in [16]-20 that such backgrounds can lead to phenomenologically interesting gauge groups, and it is therefore of interest to understand more completely the full low energy supergravity in such backgrounds. While several aspects of the effective Lagrangian have been worked out in [3, [4, [16]- 20, ,51, 52 (for a review see 45] ) the Lagrangian is extremely complicated, and many details remain to be understood more thoroughly. The present paper derives some further aspects of the low energy Lagrangian. Our main result is a formula for the potential energy for the moduli fields, valid in certain regions of moduli space. The detailed expression is given in eq. (100) et. seq. below, for the case when there is a single 5 -brane insertion and $h^{1,1}(\mathcal{X})=1$. Since the derivation is rather long we explain here a few of the ingredients of this formula.

The chiral scalars in $d=4$ supergravity take values in a target space which is KählerHodge. These fields correspond to moduli for the Calabi-Yau metric on $\mathcal{X}$, moduli for the instanton gauge field along $\mathcal{X}$, and moduli for the positions of the M5 branes along the

interval. In addition there are chiral fields charged under the gauge group $H$ left unbroken by the $E_{8} \times E_{8}$ instanton. These will generically be denoted by $C^{I}$.

The superpotential $W$ is a section of a line bundle on the Kahler-Hodge target space for the chiral scalars. There are several sources for the superpotential in the effective supergravity. First of all, there is a perturbative term, cubic in the scalars $C^{I}$. In addition, there are 
several sources of nonperturbative effects. Some of these, such as heterotic worldsheet instantons, gluino condensation, and M5 instantons (wrapping $\mathcal{X}$ ) have been studied in many previous papers [6]-15]. The inclusion of the effects of open membrane instantons, which have not been studied as thoroughly, is the main focus of this paper.

There are three kinds of open membrane effects we must consider, since M2 branes can end both on M5-branes, or on the boundary "M9 brane" [28, 29]. Membranes stretching between the boundary M9-branes are the $M$-theory versions of heterotic worldsheet instantons, and as such have been studied in the context of $(0,2)$ compactifications of heterotic string backgrounds [13, 14]. It is well-known that such effects often sum to zero, e.g., in backgrounds admitting a description by a linear sigma model [21, 22, 23]. The mechanism by which these contributions vanish is that a given homology class can contain many different holomorphic curves in $\mathcal{X}$. The instanton action depends only on the homology class, but the prefactor depends on the curve, and the sum of instanton amplitudes can, and often really does, vanish, as can already be seen in the case of the quintic. By contrast, the M2 instantons stretching between M5 and M9, or between M5 and M5 must wrap the particular holomorphic curve $\Sigma$ already wrapped by the M5 brane. This is obvious for the part of the membrane worldvolume ending on the 5-brane. A study of the conditions for the supersymmetric instanton (based on [7]) reveals that the membrane must have a direct product structure $\Sigma \times I$ where $I$ is an interval and $\Sigma \subset \mathcal{X}$ is a holomorphic curve. (The detailed argument is given in section 3 below.) Consequently, if $\Sigma$ is a rigid holomorphic curve in $\mathcal{X}$ there will be no sum over instantons, and no integral over the moduli space for the curve. Moreover, if $\Sigma$ is a rational curve there will be precisely two fermion zeromodes and the fermion 2-point function determining the superpotential will be nonzero. (Our calculation of the induced superpotential uses the technique discussed in [6, 7, 8].)

The backgrounds we study are in a regime of $M$-theory where we can do systematic expansions in the long wavelength expansion. It follows from [1, 2, 3] that this is an expansion in $R / V^{2 / 3}$ where $R$ is the length of the interval $S^{1} / Z_{2}$ and $V$ is the volume of $\mathcal{X}$ in 11 dimensional Planck units. We therefore assume $R / V^{2 / 3} \ll 1$. Now, gluino condensation and 5 -brane instanton effects contribute terms of order $\Delta W \sim \exp \left[-c_{1} V\right]$ to the superpotential $W$, where $c_{1}$ is of order 1 . By contrast, open membrane effects contribute terms of order $\Delta W \sim \exp \left[-c_{2} R V^{1 / 3}\right]$ where $c_{2}$ is of order 1 (or smaller). Thus, in the backgrounds under 
study in this paper, open membrane instantons are the leading source of nonperturbative effects.

Our goal is to understand the physics of the moduli in heterotic M-theory, so we need the potential, rather than just the superpotential. The potential energy for scalars in $d=$ $4, N=1$ supergravity is given by the famous formula 40, 39

$$
\left(\kappa_{4}\right)^{4} U=e^{K}\left(K^{i \bar{j}} D_{i} W \overline{D_{j} W}-3 W \bar{W}\right)+U_{D}
$$

where $2 \kappa_{4}^{2}=16 \pi G_{N}$ is the (four-dimensional) Newton constant, $K$ is the Kähler potential, $D_{i} W=\partial_{i} W+\partial_{i} K W$ is the covariant derivative, and $U_{D}$ are "D-terms" for charged scalars $\sim \sum_{a}\left(\bar{C} T^{a} C\right)^{2}$

The potential (11) is extremely complicated. Moreover, $K$ is only approximately known only in some regions of moduli space. We are therefore forced to consider perturbation expansions in several quantities. First, we will expand in two dimensionless parameters

$$
\mathcal{E}^{e f f} \sim R / V^{2 / 3} \ll 1 \quad \mathcal{E}_{R}^{e f f} \sim V^{1 / 6} / R \ll 1
$$

which are necessary for the validity of the geometrical 11-dimensional picture (more precise formulae appear in eq. (11) below). Note that these imply that $V \gg 1$ and $R \gg 1$, and that the length of the interval is much larger than the scale set by $\mathcal{X}$. In addition we must expand in powers of the charged scalars $C^{I}$. The superpotential is a sum of two terms $W=W_{\text {pert }}+W_{\text {nonpert }}$, where $W_{\text {pert }}$ is a cubic expression in the charged scalars $C^{I}$ with coefficients that are functions of the complex structure and bundle moduli. We can organize the terms according to whether they are order 0,1 , or 2 in $W_{\text {nonpert }}$ :

$$
\left(\kappa_{4}\right)^{4} U=\left(U_{0}+U_{1}+U_{2}\right)
$$

We will now describe the leading expressions for the three terms in (3) in the case of a Calabi-Yau $\mathcal{X}$ with $h^{1,1}(\mathcal{X})=1$ together with a single 5-brane, inserted at $x$, where $0 \leq x \leq 1$ labels the position of the 5-brane along the M-theory interval. In addition to the charged scalars $C^{I}$ the relevant chiral superfields are the "volume superfield" $S=V+i \sigma$, which determines the GUT coupling, the "Kähler superfield" $T=R a+i \chi$, where $a$ is the Kähler modulus for $\mathcal{X}$ (hence $V \sim a^{3}$ ), and the "position superfield" $Z=\operatorname{Rax}+i \alpha$ for the 5-brane. The fields $\sigma, \chi$ and $\alpha$ are axions. 
The first term, $U_{0}$, in (3) begins with the perturbative contribution to the potential. The leading order expression in an expansion in the charged scalars is a positive semidefinite quartic form:

$$
U_{0}=\frac{1}{V J^{2}} U_{I \bar{J} K \bar{L}} C^{I} \bar{C}^{\bar{J}} C^{K} \bar{C}^{\bar{L}}\left(1+\mathcal{O}\left(\frac{C^{2}}{J}, \mathcal{E}^{e f f}, \mathcal{E}_{R}^{e f f}\right)\right)
$$

Here $J:=R a$. The coefficients $U_{I \bar{J} K \bar{L}}$ are functions of the complex structure and bundle moduli. We will give precise formulae for them, but will not be very explicit about their behavior.

The leading contribution to the second term in (3) is a one-instanton term resulting from cross terms between the perturbative and nonperturbative superpotentials. We find that the single instanton contribution has the form

$$
U_{1}=\frac{(1-x)}{V J^{2}}\left\{e^{-J x} \operatorname{Re}\left[U_{I J K} C^{I} C^{J} C^{K} e^{i \alpha}\right]-e^{-J(1-x)} \operatorname{Re}\left[U_{I J K} C^{I} C^{J} C^{K} e^{i(\chi-\alpha)}\right]\right\}+\cdots
$$

The coefficients $U_{I J K}$ are functions only of the complex structure and bundle moduli.

Finally, the third term $U_{2}$ in (3) begins with a 2-instanton effect

$$
\begin{gathered}
U_{2}=\frac{E}{J^{2}}\left\{e^{-2 J x}+e^{-2 J(1-x)}-2 e^{-J} \cos (2 \alpha-\chi)\right. \\
\left.+\frac{2 J}{3 V}(1-2 x) e^{-2 J(1-x)}+\frac{4 J x}{3 V} e^{-J} \cos (2 \alpha-\chi)+\cdots\right\}\left(1+\mathcal{O}\left(\frac{C^{2}}{J}, \mathcal{E}^{\text {eff }}, \mathcal{E}_{R}^{e f f}\right)\right)
\end{gathered}
$$

where $E$ is a positive definite function that depends only on the complex structure and bundle moduli. (We have kept some subleading terms in the second line. The reason for this is explained in detail in sections 5.4 and 5.5.)

A precise characterization of the region of validity of the above potential is given in section 5.4 below. The strongest constraints on the region of validity come from our ignorance of the exact Kähler potential. It is also important to bear in mind that the coefficients of the higher order terms in the expansion in $\frac{|C|^{2}}{J}, \mathcal{E}^{e f f}, \mathcal{E}_{R}^{e f f}$ are functions of the complex structure and bundle moduli. If these coefficients become singular somewhere in the moduli space then these "higher order" terms will dominate the physics. Our working assumption is that we are at a generic smooth point in bundle and complex structure moduli space.

Having determined the leading nonperturbative effects, and thereby the potential energy, we investigate briefly some of the resulting classical dynamics on moduli space, at a somewhat heuristic level. Although the M5 branes wrap all of spacetime, thanks to the central 
term in the superalgebra, their positions along the M-theory interval are in fact dynamical variables. In the regions where we can trust our answer we find two kinds of instabilities in the compactification, depending on whether the effects of vevs of the charged scalars $C^{I}$ are important or not. When the charged scalar vevs are important, the leading $x$-dependent effect is a one-instanton effect. The axions will evolve to produce an attractive force between the M5 brane and the nearest M9-wall. This could possibly be interpreted as a consequence of the Witten effect: the axions evolve and continuously change an effective brane charge in order to produce "the most attractive channel," in particular producing an attraction between the 5-brane and the boundary. It would be interesting to understand the physics of this effect more fully.

The above discussion is valid for $J x \gg 1$. As the five-brane moves towards the wall the approximations break down. The limit $x \rightarrow 0$ is extremely interesting and is related to the chirality-changing transitions discussed in [59, 44]. In order to study this limit one needs a multiple cover formula for the membrane instantons. This is discussed in section six below. We make some educated guesses and conclude that the physics depends on the (unknown) details of the covering formula.

A second kind of instability occurs when charged scalar vevs are small or zero. In this case the potential has a local minimum in $x$ at $x=\frac{1}{2}$. The value of $U$ at such points is small and of the form

$$
U \sim \gamma \frac{e^{-J}}{J V}
$$

where $\gamma$ is a positive function of the complex structure and bundle moduli. The M2 branes lead to a repulsive interaction between the M5-brane and the M9-brane which induces decompactification of both the M-theory radius and the Calabi-Yau, while the M5-brane moves to the middle of the interval. Of course, in this instability new light modes appear as the theory becomes five-dimensional, and we should describe a matching to a description in terms of five-dimensional supergravity. (As the M5 moves to the middle of the interval there is a balancing of forces from the two boundaries and the leading terms in $U_{2}$ vanish. This is why we must include the subleading terms.)

The second kind of instability is an 11-dimensional manifestation of the Dine-Seiberg problem; it is hardly unexpected, and in the case of the standard embedding similar insta- 
bilities have already been pointed out by Banks and Dine in [4]. Nevertheless, it is interesting to note that in the 10-dimensional Dine-Seiberg instability the size of the M-theory interval $S^{1} / Z_{2}$ tends to shrink. There are thus different asymptotic regions of moduli space with qualitatively different dynamics, and hence different "basins of attraction" for the classical evolution of the moduli. One consequence is that there must be nontrivial stationary points for the potential in the middle of moduli space. The precise nature of such stationary points is of great interest, but remains out of reach so long as we cannot derive the Kähler potential in the interior of moduli space in a controlled approximation.

The paper is organized as follows. In section two we review briefly the $M$-theory geometry corresponding to strongly coupled $E_{8} \times E_{8}$ heterotic strings with "nonstandard embedding." In section three we study supersymmetric M2-brane instantons in $\mathcal{X} \times S^{1} / Z_{2}$. In section four we derive the formula for the contribution to the superpotential from M2 instantons. In section five we find the potential and specify the region where we can trust it for the simplest case of a Calabi-Yau with $h^{(1,1)}=1$. In section six we discuss the multiple covering formula and its relevance to chirality-changing transitions. In section seven we generalize the result to the case of $\mathrm{N} 5$-branes on the interval. The final section contains a discussion of some possible extensions of the present work.

We have been informed that the effects of open membranes in heterotic M-theory are also being investigated independently by B.Ovrut, E.Lima and R.Reinbacher.

\section{Review of heterotic M-theory background with M5- branes on the interval}

In this section we review some of the results of ([3, 幽, 16] - 20, 445]) which are needed for our subsequent computations.

Our conventions for the Lagrangian of 11D SUGRA are set by the Lagrangian:

$$
2 \kappa_{11}^{2} S_{11 D}=-\int e R-\frac{1}{2} \int G_{4} \wedge * G_{4}-\frac{1}{6} \int C_{3} \wedge G_{4} \wedge G_{4}+\ldots
$$

where $G_{M N P Q}=4 \partial_{[M} C_{N P Q]}$. 円

\footnotetext{
${ }^{1}$ We have a different normalization of fields compared to (16]). $G_{M N P Q}^{\text {here }}=\sqrt{2} G_{M N P Q}, C_{M N P}^{\text {here }}=$
} 
The Lagrangian of the boundary $E 8 \times E 8$ theory is given by

$$
2 \kappa_{11}^{2} S_{Y M}=-\frac{1}{4 \pi}\left(\frac{\kappa_{11}}{4 \pi}\right)^{\frac{2}{3}} \int_{M_{1}^{10}} \sqrt{-g} \operatorname{tr}\left(F^{(1)}\right)^{2}-\frac{1}{4 \pi}\left(\frac{\kappa_{11}}{4 \pi}\right)^{\frac{2}{3}} \int_{M_{2}^{10}} \sqrt{-g} \operatorname{tr}\left(F^{(2)}\right)^{2}
$$

where $F^{(1,2)}$ are the field strengths of the two $E_{8}$ gauge fields, to leading order in a longwavelength expansion. In the above action and below $t r$ means $\frac{1}{30}$ of the trace in the adjoint of $E_{8}$.

We begin by describing the background solution of $M$-theory on $R^{4} \times \mathcal{X} \times S^{1} / Z_{2}$. Our coordinates on $R^{4}$ are $x^{\mu}, \mu=1, \ldots, 4$. Complex coordinates along $\mathcal{X}$ have indices $m, \bar{m}=$ $1, \ldots, 3$. The factor $S^{1} / Z_{2}$ in spacetime has coordinate $X^{11}$. In addition it will be convenient to set $X^{11}=\pi \rho y$ where $y$ is a dimensionless coordinate $0 \leq y \leq 1$, and $\rho$ is a dimensionful constant which sets a scale.

We must now specify the metric, four-form $G_{4}$, and boundary Yang-Mills fields. In order to write the background metric we introduce a basis of harmonic $(1,1)$ forms on $\mathcal{X}$, $\omega_{i}, i=1, \ldots, h^{1,1}$ and denote the Kahler form on $\mathcal{X}$ by $\omega=a^{i} \omega_{i}$. Then, the background metric is a deformation of a metric of the form

$$
d s_{11}^{2}=V^{-1} R^{-1} g_{\mu \nu} d x^{\mu} d x^{\nu}+R^{2}\left(d X^{11}\right)^{2}-2 i \omega_{m \bar{m}} d x^{m} d x^{\bar{m}}
$$

In this formula $R$ is dimensionless and $R \rho$ is the orbifold radius. Similarly, we introduce a fiducial, dimensionful, volume $v$ for $\mathcal{X}$, and the volume of $\mathcal{X}$ in the metric (9) defines the dimensionless parameter $V$ by $V v:=\frac{1}{3 !} \int_{\mathcal{X}} \omega^{3}$. We will make a convenient choice of $\rho, v$ in eq. (12) below; they will be of the order of $l_{11}, l_{11}^{6}$ and are independent of moduli. Because of the Weyl-rescaling in the first term in (9), $g_{\mu \nu}$ is the four-dimensional Einstein metric and the four-dimensional Newton constant is given by

$$
\frac{1}{\kappa_{4}^{2}}=\frac{2 \pi \rho v}{\kappa_{11}^{2}}
$$

As we have mentioned, the actual metric we will use is a deformation of eq. (9), and is only known to first order in a power series in two dimensionless expansion parameters

$$
\mathcal{E}^{e f f}=\frac{\epsilon R}{V^{\frac{2}{3}}} \ll 1, \quad \mathcal{E}_{R}^{e f f}=\frac{\epsilon_{R} V^{\frac{1}{6}}}{R} \ll 1
$$

$6 \sqrt{2} C \frac{16 \mid}{M N}$. We use the convention $2 \kappa_{11}^{2}=(2 \pi)^{8}\left(M_{11}\right)^{-9}$, and define the 11-dimensional Planck length by $l_{11}=1 / M_{11}$. Our signature is mostly plus. 
where we choose the constants

$$
\epsilon=\left(\frac{\kappa_{11}}{4 \pi}\right)^{\frac{2}{3}} \frac{2 \pi^{2} \rho}{v^{\frac{2}{3}}}=2, \quad \epsilon_{R}=\frac{v^{\frac{1}{6}}}{\pi \rho}=\left(\frac{\pi}{2}\right)^{\frac{1}{2}}
$$

in order to simplify the normalization of the fields in the effective Lagrangian.

The above inequalities (11) state, firstly, that the distortion of the background from (9) is small, and secondly that the interval is much larger than the length scale of $\mathcal{X}$. These expansion parameters can be related to the GUT scale and the 4-dimensional Newton constant [3, 4]. In our conventions the unified coupling $\alpha_{\mathrm{GUT}} \sim\left(\mathcal{E}^{e f f} \mathcal{E}_{R}^{\text {eff }}\right)^{2} \sim 1 / V$, while $\left(M_{\mathrm{GUT}} \kappa_{4}\right)^{2} \sim\left(\mathcal{E}^{e f f}\right)^{3}\left(\mathcal{E}_{R}^{e f f}\right)^{4} \sim 1 /\left(R V^{4 / 3}\right)$ determines the GUT scale in terms of the Newton constant. The latter formula follows by computing masses of gauge bosons and scalars associated with typical mechanisms of spontaneous symmetry breaking. [ Unfortunately, it turns out that when we use the experimentally measured values of $\alpha_{\mathrm{GUT}}, M_{\mathrm{GUT}}$ and $\kappa_{4}$ the above expansion is not necessarily a good approximation. As discussed in [3, 4, 5, 16, 53], the experimentally measured values determine $\mathcal{E}_{R}^{e f f} \ll \mathcal{E}^{e f f}=O(1)$. Nevertheless, our focus in this paper is on a systematic and controlled computation of nonperturbative effects; the restriction (11) is necessary since heterotic M-theory is only known as an effective theory to order $\left(\kappa_{11}\right)^{\frac{2}{3}}$, and for this reason we will adopt it.

To lowest order in the expansion parameter the metric for the background takes the form

$$
\begin{gathered}
d s_{11}^{2}=V^{-1} R^{-1}\left(1+\frac{B}{6}\right) g_{\mu \nu} d x^{\mu} d x^{\nu}+R^{2}\left(1-\frac{2 B}{3}\right)\left(d x^{11}\right)^{2}-2 i J_{m \bar{m}} d x^{m} d x^{\bar{m}}, \\
J_{m \bar{m}}=\omega_{m \bar{m}}+\left(B_{m \bar{m}}-\frac{1}{3} \omega_{m \bar{m}} B\right), \quad B=2 \omega^{m \bar{m}} B_{m \bar{m}},
\end{gathered}
$$

The deformation of the background is described by the $(1,1)$ form $B_{n \bar{m}}$. In order to write it explicitly we must now introduce the M5 branes.

The backgrounds we study preserve $N=1$ supersymmetry. Therefore the 5 -branes wrap a product of spacetime and a holomorphic curve in $\mathcal{X}$. If there are $N$ 5-branes they will therefore have definite locations at $y=x_{k}, k=1, \ldots, N$ along the interval. The $k^{\text {th }} 5$-brane wraps a curve $\Sigma^{(k)}$ in $\mathcal{X}$ whose homology class may be expressed as $\left[\Sigma^{(k)}\right]=\beta_{i}^{(k)}\left[\Sigma_{2}^{i}\right]$ where $\left[\Sigma_{2}^{i}\right]$ is an integral basis of $H_{2}(X, \mathbf{Z})$, and $\beta_{i}^{(k)}$ is a collection of nonnegative integers. These

\footnotetext{
${ }^{2}$ We take $v=8 \pi^{5} l_{11}^{6}, \quad \pi \rho=2 \pi^{\frac{1}{3}} l_{11}$ to have $\epsilon=2, \epsilon_{R}{ }^{2}=\frac{1}{2} \pi$

${ }^{3}$ We thank T. Banks for very helpful discussions on this point.
} 
integers are constrained by anomaly cancellation. Each of the M9 branes carries an $E_{8}$ vector bundle $V_{1}, V_{2}$, and to each bundle we associate a degree four integral characteristic class $c_{2}\left(V_{i}\right)$. Identifying $H_{2}(\mathcal{X} ; Z)$ with $H^{4}(\mathcal{X} ; Z)$ via Poincaré duality we may define

$$
c_{2}\left(V_{1}\right)-\frac{1}{2} c_{2}(T X)=\beta_{i}^{(0)}\left[\Sigma_{2}^{i}\right] \quad c_{2}\left(V_{2}\right)-\frac{1}{2} c_{2}(T X)=\beta_{i}^{(N+1)}\left[\Sigma_{2}^{i}\right]
$$

The anomaly cancellation condition is then

$$
\sum_{n=0}^{N+1} \beta_{i}^{(n)}=0
$$

In terms of the above data, the formula for $B_{m \bar{m}}$ on the interval $\left(x_{n}, x_{n+1}\right), \quad n=0, \ldots, N$ is given by

$$
B_{m \bar{m}}=\frac{2 R}{V} b_{i} \omega_{m \bar{m}}^{i}, \quad b_{j}(y)=\sum_{k=0}^{n} \beta_{j}^{(k)}\left(y-x_{k}\right)-\frac{1}{2} \xi_{j}, \quad \xi_{j}=\sum_{k=0}^{N+1}\left(1-x_{k}\right)^{2} \beta_{j}^{(k)},
$$

where $x_{0}=0, x_{N+1}=1$ and the index $i$ is raised with the inverse of the metric on the moduli space of Kahler structures on $\mathcal{X}$ :

$$
G_{i j}=\frac{1}{2 v V} \int_{\mathcal{X}} \omega_{i} \wedge\left(* \omega_{j}\right)=-\frac{1}{2} \partial_{i} \partial_{j} \ln \left(d_{i_{1} i_{2} i_{3}} a^{i_{1}} a^{i_{2}} a^{i_{3}}\right)
$$

with

$$
d_{i_{1} i_{2} i_{3}}=\int_{\mathcal{X}} \omega_{i_{1}} \wedge \omega_{i_{2}} \wedge \omega_{i_{3}}
$$

The choice of integration constant in the solution (16) fixes $V v$ to be equal to the volume of $\mathcal{X}$ averaged along the interval (to lowest order in $\mathcal{E}^{e f f}$ ).

The flux of the 4-form $G_{4}$ is also given in terms of $B$ :

$$
G_{M N P Q}=\frac{1}{2} \epsilon_{M N P Q E F} \partial_{11} B^{E F}
$$

Note that it is discontinuous across the positions of the 5-branes.

Finally, we need to specify the $E_{8}$ gauge bundles $V_{1}$ and $V_{2}$. For simplicity we will follow [17] and take the bundle $V_{2}$ at $y=1$ to be the trivial bundle. Accordingly, there is a "hidden sector" at $y=1$ with unbroken $E_{8}$ gauge group. The bundle $V_{1}$ at $y=0$ has an instanton whose holonomy lives in a subgroup $G \subset E_{8}$. The unbroken gauge symmetry is the commutant $H$ of $G$ in $E_{8}$. It is straightforward to extend our formulae to the case when both $V_{1}$ and $V_{2}$ are non-trivial bundles. 
When we compactify M-theory on the above background, the physics at distances large compared to the M-theory interval is described by an effective $d=4, N=1$ supergravity theory. We now list the massless fields corresponding to small fluctuations around the above background. In addition to the superYang-Mills and supergravity multiplets there are a number of massless chiral scalar fields. To begin with, there are chiral superfields neutral under four dimensional gauge group $H$. These are:

$$
\begin{gathered}
T^{i}=R a^{i}+i \chi^{i}, \\
S=V+i \sigma \\
Z_{n}=R\left(\beta_{i}^{(n)} a^{i}\right) x_{n}-i\left[\mathcal{A}_{n}\left(\beta_{i}^{(n)} a^{i}\right)-x_{n}\left(\beta_{i}^{(n)} \chi^{i}\right)\right]
\end{gathered}
$$

where

$$
C_{m \bar{m} 11}=\chi^{i} \omega_{i, m \bar{m}}, \quad i=1, \ldots, h^{1,1}, \quad m, \bar{m}=1, \ldots, 3
$$

$\sigma$ is a scalar dual to $C_{\mu \nu 11}$

$$
3 \partial_{[\mu} C_{\nu \rho] 11}=V^{-2} \epsilon_{\mu \nu \rho \lambda} \partial^{\lambda} \sigma
$$

and $Z_{n}$ is a holomorphic coordinate constructed out of the position $x_{n}$ of the n-th 5-brane on the interval. The scalar $\mathcal{A}_{n}$ originates from the KK reduction of the 2 -form living on the n-th 5-brane

$$
A_{n}^{(2)}=\pi \rho \mathcal{A}_{n} f_{n}^{*}(\omega)
$$

We have included the factor $\pi \rho$ in the above formula to make $\mathcal{A}_{n}$ dimensionless. In eq.(23) $f_{n}^{*}(\omega)$ is the pullback of the Kahler form to the cycle $\Sigma_{2}^{(n)}$. We denote by $f_{n}$ the holomorphic embedding of the curve $\Sigma_{2}^{(n)}$ in $\mathcal{X}$. The pullback of each of the basis forms $f_{n}^{*}\left(\omega_{i}\right)$ is proportional to the pullback of the Kahler form $\omega$

$$
f_{n}^{*}\left(\omega_{i}\right)=\frac{\beta_{i}^{(n)}}{\left(\beta_{j}^{(n)} a^{j}\right)} f_{n}^{*}(\omega), \quad \int_{\Sigma_{2}^{(n)}} f_{n}^{*}\left(\omega_{i}\right)=v^{\frac{1}{3}} \beta_{i}^{(n)} .
$$

Finally, there are chiral multiplets charged under the unbroken gauge group $H$. Thanks to the Donaldson-Uhlenbeck-Yau theorem massless modes from small fluctuations of the gauge field can be associated with holomorphic deformations of holomorphic bundles on $\mathcal{X}$. The small fluctuations are parametrized by the space $H_{\bar{\partial}}^{0,1}(X, V)$ where $V$ is the gauge bundle in the 248. We assume the holonomy of the instanton is in $G$ so the gauge bundle 
decomposes as $V=\oplus W_{\mathcal{R}} \otimes V_{\mathcal{S}}$ corresponding to the decomposition of the adjoint of $E_{8}$ under the embedding $H \times G \subset E_{8}$ :

$$
248=\oplus \mathcal{R} \otimes \mathcal{S}
$$

The charged scalars will be valued in $\oplus W_{\mathcal{R}} \otimes H_{\bar{\partial}}^{0,1}\left(X, V_{\mathcal{S}}\right)$. In order to work out the KaluzaKlein reduction we decompose the gauge field as:

$$
A_{\bar{m}}=\frac{2^{\frac{3}{2}} \pi}{\kappa_{4}} u_{\hat{I}, \bar{m}} C^{\hat{I}}, \quad \bar{m}=1,2,3,
$$

In (25) a summation is taken over the index $\hat{I}$ which labels

$$
\hat{I}=(\mathcal{R}, I, p), \quad p=1, \ldots, \operatorname{dim} \mathcal{R}, \quad I=1, \ldots \operatorname{dim} H^{1}\left(X, V_{1 \mathcal{S}}\right)
$$

The normalization factor in (25) was chosen to make the charged scalar fields $C^{\hat{I}}$ dimensionless and to normalize their kinetic term conveniently.

When writing the perturbative superpotential below it will be convenient to define

$$
u_{\hat{I}, \bar{m}}=u_{I \bar{m}}^{x} T_{x p}
$$

where $x$ is an index for a basis for the representation $\mathcal{S}$ and $u_{I \bar{m}}^{x}$ is a basis of $H^{1}\left(X, V_{1 \mathcal{S}}\right)$. The factor $T_{x p}$ is purely group-theoretic and corresponds to the generators of $E_{8}$ in the representation $\mathcal{R} \otimes \mathcal{S}$. The complex conjugate of these generators is denoted by $T^{x p}$ and the normalization is chosen such that $\operatorname{tr}\left(T_{x p} T^{y q}\right)=\delta_{x}^{y} \delta_{p}^{q}$.

We are not going to study four-dimensional gauge dynamics in this paper. This has been studied, for example, in [3, 4, 17]. For completeness, and to fix our normalizations, we also give the gauge kinetic term in the $4 \mathrm{D}$ Lagrangian

$$
S_{Y M}=-\sum_{\alpha=1}^{2} \frac{1}{64 \pi^{2}} \int_{M_{4}^{(\alpha)}} \sqrt{-g_{4}}\left(\operatorname{Ref} f^{\alpha} \operatorname{tr} F^{2}+\ldots\right.
$$

where due to the restrictions (11) on the moduli space, $\operatorname{Re} f^{\alpha}=V+O\left(\mathcal{E}^{\operatorname{eff}}\right), \quad \alpha=1,2$.

\section{M2-brane instantons in $\mathcal{X} \times S^{1} / Z_{2}$}

Open M2-branes ending on an M5 brane will play a crucial role in our calculation of the non-perturbative potential. These nonperturbative effects were first discussed in [28, 29, 42]. 
In this section we will derive the conditions for a supersymmetric open M2-brane instanton in the background described in the previous section. We will neglect the distortion of the background metric from a direct product metric in solving for the membrane configuration. This is valid in our approximation scheme.

The first step in finding the supersymmetric M2 configuration is to write the constant spinors corresponding to the supersymmetries unbroken by the background. We use a basis for the $\Gamma$-matrices in eleven dimensions of the form

$$
\begin{array}{rl}
\Gamma^{\mu}=(R V)^{\frac{1}{2}} \gamma^{\mu} \otimes \gamma^{7} \quad \Gamma^{m}=1 \otimes \gamma^{m} & \mu=1, \ldots, 4, \quad\left\{\gamma^{\mu}, \gamma^{\nu}\right\}=2 g^{\mu \nu} \\
\Gamma^{\bar{m}}=1 \otimes \gamma^{\bar{m}} \quad \Gamma^{11}=\frac{1}{R} \gamma^{5} \otimes \gamma^{7} & m, \bar{m}=1, \ldots, 3, \quad\left\{\gamma^{n}, \gamma^{\bar{m}}\right\}=2 g_{C Y}^{n \bar{m}}
\end{array}
$$

where $\left(\gamma_{m}\right)^{*}=-\gamma_{\bar{m}}=\left(\gamma_{m}\right)^{T}$ and $\gamma^{\mu}$ is a weyl-basis in $4 \mathrm{D}$.

Four dimensional anti-chiral ( chiral) spinor indices are denoted by $\alpha(\dot{\alpha})$ respectively. In this basis the surviving supersymmetry in the background $\mathcal{X} \times S^{1} / Z_{2}$ is of the form:

$$
\epsilon=\left(\epsilon^{\dot{\alpha}} \otimes \epsilon_{1}, \epsilon^{\alpha} \otimes \epsilon_{2}\right)
$$

where $\epsilon^{\dot{\alpha}}, \epsilon^{\alpha}$ are constant spinors on $R^{4} \times S^{1} / Z_{2}$ and $\epsilon_{1}\left(\epsilon_{2}=\left(\epsilon_{1}\right)^{*}\right)$ is the chiral( anti-chiral) covariantly constant spinor on $\mathcal{X}$, normalized as in [7]:

$$
\gamma_{\bar{m}} \epsilon_{1}=0, \quad \gamma_{n \bar{m}} \epsilon_{1}=i \omega_{n \bar{m}} \epsilon_{1}, \quad \gamma_{m n p} \epsilon_{1}=e^{-K} \Omega_{m n p} \epsilon_{2}, \quad \epsilon_{1}^{\dagger} \epsilon_{1}=1
$$

Here $\omega$ is the Kahler form, $\Omega$ is a holomorphic $(3,0)$ form on $\mathcal{X}$ and $K=\frac{1}{2}\left(K_{T}-K_{c p l x}\right)$ with both Kahler functions $K_{T}$ and $K_{c p l x}$ specified in section (5.2).

The surviving supersymmetry is consistent with having 5-branes wrapped over a holomorphic cycle $\Sigma \subset \mathcal{X}$, as shown in [16]. One cannot have anti-5-branes on the interval and preserve supersymmetry.

The presence of an M2-brane imposes an additional constraint on the supersymmetry parameter $\epsilon$

$$
\Gamma^{(2)} \epsilon=\epsilon
$$

where, (see for example [7]),

$$
\Gamma^{(2)}=\frac{i}{3 ! \sqrt{g}} \epsilon^{i j k} \partial_{i} X^{\hat{M}} \partial_{j} X^{\hat{N}} \partial_{k} X^{\hat{K}} \Gamma_{\hat{M} \hat{N} \hat{K}}
$$


In formula (32) $s^{i}, i=1,2,3$ are coordinates on the world-volume of the M2-brane, $X^{\hat{M}}, \hat{M}=$ $(\mu, m, \bar{m}, 11)$ are coordinates in the eleven dimensional target space and $g$ is the determinant of the induced metric on the M2-brane.

Substituting (29) into (31) we find, first of all, that spinors of type $\epsilon^{\alpha} \otimes \epsilon_{2}$ lead to

$$
\begin{aligned}
& \epsilon_{2}=\left(\frac{R}{\sqrt{g}} \epsilon^{i j k} \partial_{i} X^{m} \partial_{j} X^{\bar{n}} \partial_{k} X^{11} \omega_{m \bar{n}}\right) \epsilon_{2}+\left(\frac{i e^{-K}}{3 ! \sqrt{g}} \epsilon^{i j k} \partial_{i} X^{\bar{m}} \partial_{j} X^{\bar{n}} \partial_{k} X^{\bar{p}} \Omega_{\overline{m n p}}\right) \epsilon_{1} \\
& +\left(\frac{\epsilon^{i j k}}{\sqrt{g}} \partial_{i} X^{m} \partial_{j} X^{\bar{n}} \partial_{k} X^{\bar{p}} \omega_{m \bar{n}}\right) \gamma_{\bar{p}} \epsilon_{2}+\left(\frac{i e^{-K}}{4 \sqrt{g}} \epsilon^{i j k} \partial_{i} X^{\bar{m}} \partial_{j} X^{\bar{n}} \partial_{k} X^{11} \Omega_{\overline{m n p}} g_{C Y}^{\bar{p} q}\right) \gamma_{q} \epsilon_{1}
\end{aligned}
$$

Since the spinors $\epsilon_{1}, \epsilon_{2}, \gamma_{m} \epsilon_{1}, \gamma_{\bar{m}} \epsilon_{2}$ are linearly independent we get four equations

$$
\begin{gathered}
\partial_{i} X^{\bar{m}} \partial_{j} X^{\bar{n}} \partial_{k} X^{\bar{p}} \Omega_{\overline{m n p}}=0 \\
R \partial_{i} X^{m} \partial_{j} X^{\bar{n}} \partial_{k} X^{11} \omega_{m \bar{n}}=\sqrt{g} \epsilon_{i j k} \\
\partial_{i} X^{\bar{m}} \partial_{j} X^{\bar{n}} \partial_{k} X^{11} \Omega_{\overline{m n p}} g_{C Y}^{\bar{p} q}=0 \\
\epsilon^{i j k} \partial_{i} X^{m} \partial_{j} X^{\bar{n}} \partial_{k} X^{\bar{p}} \omega_{m \bar{n}}=0
\end{gathered}
$$

The constraints $(34,37)$ are automatically solved by the embedding

$$
X^{11}=t, \quad X^{m}(y)
$$

where $t=s^{3}$ is a coordinate along the orbifold interval and $y, \bar{y}$ are coordinates on a holomorphic 2-cycle. This is our basic instanton.

We claim that if the holomorphic curve $\Sigma \subset \mathcal{X}$ is isolated then the above membrane instanton is also. Moreover, we claim that the above instanton is the only instanton solution consistent with the boundary condition of having the M2 brane ending on $\Sigma$. Indeed, let us consider the possibility of having M2-branes starting and ending on holomorphic cycles inside $\mathcal{X}$ which differ from a direct product $\Sigma \times I$. Therefore we search for $t$-dependent embeddings $X^{m}(y, t), t \in\left[x_{1}, x_{2}\right]$ into $\mathcal{X}$. In this case equation (37) is not satisfied automatically and gives the constraint

$$
\partial_{[i} X^{m} \partial_{j]} X^{\bar{n}} \omega_{m \bar{n}}=0
$$

Taking the $i=y, j=\bar{y}$ component of this equation and evaluating it at the boundary $t=x_{1}$ or $t=x_{2}$ shows that the volume of the holomorphic cycle must be zero. 
We conclude that an open M2-brane which starts and ends on a positive volume holomorphic curve preserves some supersymmetry iff it has the direct product form $\Sigma \times I$.

One can quite analogously prove that an M2-brane which starts and ends on a holomorphic curve should have the direct product form

$$
X^{11}=-t, \quad X^{m}(y)
$$

in order to preserve the other components $\epsilon^{\dot{\alpha}} \otimes \epsilon_{1}$ of the background supersymmetry.

Note that since the M2-brane instanton must start and end on the same 2-cycle in $\mathcal{X}$

there is a requirement on the 5 -brane charges $\beta_{i}^{(n)}=\beta_{i}^{(k)}$ described in section 2 in order for there to be an M2-instanton stretched in the interval $\left[x_{n}, x_{k}\right]$.

\section{Calculation of membrane-instanton-induced super- potentials}

In this section we will give the derivation of the non-perturbative four-dimensional superpotential $\Delta W$ induced by open membranes.

We follow the procedure outlined in [7, 8]. The idea is to compute the 2-point correlation function of four-dimensional fermions with the instanton sector included in the supergravity path integral. An essential ingredient of this calcultaion is the coupling of the four-dimensional fermions to the world-volume degrees of freedom of the membrane through the so-called "membrane vertex operators." The computation of the superpotential follows from a computation of a 2-point correlation function of fermions in the four-dimensional effective theory $\langle\chi \chi\rangle_{\text {inst }}$, where $\chi$ are fermionic superpartners of $Z$. This in turn can be reduced to a membrane path integral with corresponding vertex operator insertions.

\subsection{Summary of the computation of $\Delta W$}

Since the analysis is rather long let us summarize the computation here. Most of the work is devoted to finding the vertex operator, but the end result is very simple. The membrane theory has a chiral doublet of fermions $\vartheta^{\dot{\alpha}}$ transforming in the $\mathbf{2}$ of the 4 dimensional Lorentz 
group. These couple to the chiral fermions $\chi_{\dot{\alpha}}$ in the superfield $Z$ via the vertex operator

$$
V_{\chi}=\frac{i}{2} \vartheta^{\dot{\alpha}} \chi_{\dot{\alpha}}
$$

Using the above coupling we can compute $\left\langle\chi\left(\xi_{1}\right) \chi\left(\xi_{2}\right)\right\rangle$ in an instanton sector to be

$$
\int \sqrt{-g_{4}} d^{4} \xi S_{F}\left(\xi_{1}-\xi\right) S_{F}\left(\xi_{2}-\xi\right) h \Phi \exp (-Z) .
$$

Here $\xi_{1}, \xi_{2}$ are points in four dimensions and $S_{F}$ is the 4-dimensional fermion propagator in the effective $d=4, N=1$ supergravity. This expression for the propagator is only valid for $\left(\xi_{1}-\xi\right),\left(\xi_{1}-\xi_{2}\right),\left(\xi_{2}-\xi\right) \gg l_{11}$. The integral of $\xi^{\mu}$ in eq.(40) should be regarded as an integral over the bosonic zero modes $X^{\mu}=\xi^{\mu}$ of the M2-instanton. The integral over the 2 fermion zero-modes $\vartheta^{1}, \vartheta^{2}$, on the M2-brane soaks up the $\vartheta^{\dot{\alpha}}$ from the vertex operator. There are no other zero modes because the curve $\Sigma$ is a rational curve and hence has no extra zeromodes associated with 1-forms. The prefactor $h \Phi$ stands for determinants of fluctuations in 11-dimensional supergravity together with 5-branes around the background (13,19), together with determinants associated with the degrees of freedom for the M2 instanton. While it is very complicated one can use holomorphy to extract the factor $h e^{-Z}$, which depends holomorphically on the moduli. The factor $h$ is a holomorphic section of a line bundle over complex structure moduli space and should properly be regarded as the true measure for the fermion zeromodes. In this paper we will not be very explicit about it.

We can now extract $\Delta W$ by comparing (40) with the 2-point correlation function in the effective 4D supergravity

$$
\left\langle\chi\left(\xi_{1}\right) \chi\left(\xi_{2}\right) \exp \left[\int \sqrt{g_{4}} e^{\frac{1}{2} K} \partial_{Z} \partial_{Z}(\Delta W) \bar{\chi} \bar{\chi}\right]\right\rangle_{4 D}
$$

which is equal to

$$
\int \sqrt{g_{4}} d^{4} \xi S_{F}\left(\xi_{1}-\xi\right) S_{F}\left(\xi_{2}-\xi\right) e^{\frac{1}{2} K_{0}} \partial_{Z} \partial_{Z}(\Delta W)
$$

where $K_{0}=K_{T}+K_{S}+K_{c p l x}+K_{\text {bundle }}$ and we drop corrections of the order $O\left(\mathcal{E}^{\text {eff }}, \mathcal{E}_{R}^{\text {eff }}, \frac{|C|^{2}}{R a}\right)$ to the mass term for a chiral fermion in the $4 \mathrm{D}, \mathrm{N}=1$ Lagrangian [40, 39].

Using holomorphy of the superpotential it now follows that

$$
\Delta W=h \exp (-Z), \quad \Phi=e^{\frac{1}{2} K_{0}} .
$$


For the M2-brane stretched between the 5-brane and the other 9-brane at $y=1$ analogous considerations give

$$
\Delta W=h \exp \left(-\left(\beta_{i} T^{i}-Z\right)\right)
$$

\subsection{Computation of the vertex operator}

In this section we describe the computation of the vertex operator.

The vertex operators can be found by expanding the action of the $M 2$ brane in the Mtheory background fields. The action of an M2-brane ending on an M5-brane was written in [35], using the superembedding approach of [30, 31]. In this approach the basic ingredients are:

- An (11|32) supermanifold $M$, giving the 11-dimensional supergravity background. The supercoordinates are denoted by $Z \underline{\underline{M}}=\left(X^{\hat{M}}, \Theta^{\hat{\rho}}\right)$. where $\hat{\rho}$ is an index in the irreducible spinor representation of $s o(1,10)$. Using the torsion constraints of [32 on the supervielbein one can expand an orthonormal frame for the cotangent space in powers of $\Theta$. The expansion at low orders in $\Theta$ has been worked out in [8, 34, 9].

It is convenient to introduce the notation for the vielbein:

$$
E^{\underline{A}}(Z)=d Z^{\underline{M}} E_{\underline{M}} \underline{A}=\left(E^{\underline{a}}, E^{\underline{\alpha}}\right)
$$

where in the second equality we have separated bosonic and fermionic cotangent vectors.

- A (6|16) supermanifold $\mathcal{M}$ describing the world-volume of the M5-brane. We denote supercoordinates on the worldvolume by $z^{M}=\left(y^{M}, \vartheta^{\rho}\right)$ and a cotangent frame on $\mathcal{M}$ by $e^{A}(z)$. A decomposition of the frame analogous to (45) is given by

$$
e^{A}(z)=d z^{M} e_{M}^{A}=\left(e^{a}, e^{\beta q}\right)
$$

The index $a=0,1, \ldots, 5$ is the index of the vector representation of $s o(1,5)$, while $\beta$ and $q$ are the indices of irreducible spinor representations of $s o(1,5)$ and $s o(5)$, respectively.

- A (3|0) manifold $\Sigma$, to be identified with the membrane worldvolume. The boundaries of $\Sigma$ lie inside $\mathcal{M}$ or in $\partial M$. We denote the coordinates on $\Sigma$ by $s^{i}, i, \ldots, 3$. Coordinates on the boundary surface are denoted by $\sigma^{r}, r=1,2$. 
The relation of the pullback of the 11-dimensional supervielbein to the 5-brane to the supervielbein of the 5 -brane itself defines the "embedding matrices" $E_{A} \underline{A}$ via the equation

$$
f^{*}\left(E^{\underline{A}}\right)=e^{A} E_{A} \underline{A}
$$

One may solve for these matrices in terms of the vielbeins

$$
E_{A} \underline{A}=e_{A}{ }^{M} \partial_{M} Z^{\underline{M}} E_{\underline{M}} \underline{A} .
$$

The basic superembedding condition then says that

$$
E_{\text {fermionic }}^{\text {bosonic }}=E_{\beta q}{ }^{a}=0 \text {. }
$$

This simple equation is extremely powerful, it leads to a complete set of covariant equations of motion for the 5-brane [30, 31].

The action of an M2-brane ending on an M5-brane, in Euclidean signature, is 46, 35]

$$
S_{M 2}=\tau_{M 2} \int_{\Sigma} d^{3} s\left[\sqrt{{\operatorname{det} g_{i j}}}+i f^{*} \mathbf{C}^{(3)}\right]-i \tau_{M 2} \int_{\partial \Sigma} d^{2} \sigma \phi^{*} B^{(2)}
$$

Here $\tau_{M 2}=\frac{1}{(2 \pi)^{2}} M_{11}^{3}$ is the M2-brane tension. Also, $B^{(2)}$ is the super 2-form living on $\mathcal{M}$ while $\mathbf{C}^{(3)}$ is the super 3 -form living on the target superspace $M$. The pullback in eq.(50) under the embedding $f: s^{i} \rightarrow Z \underline{M}$ is

$$
f^{*} \mathbf{C}^{(3)}=\frac{1}{3 !} \partial_{i} Z^{\underline{M}} \partial_{j} Z^{\underline{N}} \partial_{k} Z^{\underline{P}} \mathbf{C}_{\underline{M N P}}^{(3)} d s^{i} \wedge d s^{j} \wedge d s^{k}
$$

while the pullback under the embedding $\phi: \sigma^{r} \rightarrow z^{M}$ is

$$
\phi^{*} B^{(2)}=\frac{1}{2} \partial_{r} z^{M} \partial_{s} z^{N} B_{M N}^{(2)} d \sigma^{r} \wedge d \sigma^{s}
$$

We specialize the action (50) to the case of an M2-brane stretched between $y=0$ and $y=x$ in the background described in section 2 . The membrane is a product $\Sigma \times[0, x]$ so it is convenient to define coordinates on the membrane $s^{i}=(t, \sigma, \bar{\sigma})$ where $t$ is a coordinate on the interval and $\sigma$ is a holomorphic coordinate along the curve $\Sigma$. The embedding coordinates of $\Sigma$ into (11|32) superspace

$$
Z^{\underline{M}}(s)=\left(X_{3,11}^{\hat{M}}(s), \Theta_{3,11}(s)\right)
$$


have the following structure. First, the interval coordinate

$$
X_{3,11}^{11}(s)=\pi \rho\left(t+\frac{i}{2} \Theta_{3,11} \Gamma^{11} \bar{\Theta}_{3,11}\right)
$$

has an important correction quadratic in fermions, while the coordinates

$$
X_{3,11}^{m}(s)=X^{m}(\sigma), \quad X_{3,11}^{\bar{m}}(s)=X^{\bar{m}}(\bar{\sigma})
$$

describe the holomorphic embedding. The coordinate $X_{3,11}^{\mu}(s)$ is unconstrained. The fermions $\Theta_{3,11}(s)$ satisfy the physical gauge condition

$$
\Gamma^{(2)} \Theta_{3,11}(s)=-\Theta_{3,11}(s)
$$

We have omitted the coordinates describing fluctuations of the membrane within $\mathcal{X}$ since we will restrict our consideration to an isolated curve $\Sigma$ and hence these degrees of freedom will be massive.

The origin of the correction in (52) is continuity of embedding coordinates in superspace. That is, the embedding of the membrane into 11-dimensional superspace $(3 \mid 0) \rightarrow(11 \mid 32)$ must agree, on the boundary, with the embedding of the 5-brane into superspace $(6 \mid 16) \rightarrow$ (11|32) since the membrane ends on the 5-brane in superspace. We now derive this condition in more detail.

We choose bosonic coordinates on the M5-brane as $y^{M}=\left(y^{\mu}, y, \bar{y}\right)$ where $y^{\mu}$ are real and $y$ is complex, and consider the static embedding of the boundary of the M2-brane into the M5-brane

$$
\phi: y=\sigma, \bar{y}=\bar{\sigma}
$$

The superembedding $(6 \mid 16) \rightarrow(11 \mid 32)$ is described by superfields

$$
Z^{\underline{M}}=\left(X^{\hat{M}}\left(z^{M}\right), \Theta\left(z^{M}\right)\right)
$$

Small fluctuations around static gauge are described by embeddings

$$
X^{\hat{M}}=\left(y^{m}, X^{m^{\prime}}(y, \vartheta)\right), \quad \Theta=(\vartheta, \psi(y, \vartheta))
$$

where $\vartheta$ is a fermionic coordinate in the (6|16) superspace. The superfields $X^{m^{\prime}}(y, \vartheta)$ and $\psi(y, \vartheta)$ have as their $\vartheta=0$ component bosonic fluctuations transverse to the worldvolume 
of the M5-brane $X^{m^{\prime}}\left(y^{M}\right)$ and physical fermions on the M5-brane $\psi\left(y^{M}\right)$ respectively. $m^{\prime}$ here denotes bosonic indices of coordinates transverse to the M5-brane.

As was discussed, for example, in [31, the basic superembedding condition (49) imposes a relation on the superfields $X^{m^{\prime}}(y, \vartheta)$ and $\psi(y, \vartheta)$, such that

$$
X^{m^{\prime}}\left(y^{M}, \vartheta\right)=X^{m^{\prime}}\left(y^{M}\right)+i \vartheta \Gamma^{m^{\prime}} \psi(y)+\ldots
$$

In particular, the superfield $X^{11}$ up to linear order in $\vartheta$ is

$$
X_{6,11}^{11}=\pi \rho\left(x+i \vartheta \Gamma^{11} \bar{\psi}+\ldots\right)
$$

Recall that we introduced the factor $\pi \rho$ to make $x$ dimensionless.

In the geometry of $\mathcal{X}$, the spinors $\vartheta$ and $\psi$ can be decomposed as

$$
\begin{aligned}
& \vartheta=\left\{\vartheta^{\dot{\alpha}} \otimes \epsilon_{1}, \vartheta^{\alpha} \otimes \epsilon_{2}\right\} . \\
& \psi=\left\{\psi^{\alpha} \otimes \epsilon_{1}, \psi^{\dot{\alpha}} \otimes \epsilon_{2}\right\},
\end{aligned}
$$

Out of the 16-component spinors we only keep those components given by the covariantly constant spinor along $\mathcal{X}$. There are other physical degrees of freedom in the spinor $\psi$, but since we are considering a rigid curve in $\mathcal{X}$ only the above components lead to massless degrees of freedom.

In Euclidean space equation (58) becomes

$$
X_{6,11}^{11}=\pi \rho\left(x-\frac{i}{R}\left(\vartheta^{\dot{\alpha}} \psi_{\dot{\alpha}}+\vartheta^{\alpha} \psi_{\alpha}\right)\right)
$$

where now chiral and anti-chiral spinors are independent from each other. (To give the meaning to the fermionic bilinears in Euclidean space, we first define them in Minkowskii space, where

$$
\vartheta_{\dot{\alpha}}=\left(\vartheta^{\alpha}\right)^{*}, \quad \psi_{\dot{\alpha}}=\left(\psi^{\alpha}\right)^{*}, \quad \gamma^{0}=\left(\begin{array}{cc}
0 & 1 \\
1 & 0
\end{array}\right)
$$

where the spinors indices are lowered via $\vartheta_{\dot{\alpha}}:=\varepsilon_{\dot{\alpha} \dot{\beta}} \vartheta^{\dot{\beta}}$. Then we continue to Euclidean signature by dropping the reality conditions in eq.(62).)

From eq.(52) and eq.(61) we see that $X_{3,11}^{11}$ and $X_{6,11}^{11}$ match each other at the boundary of the M2-brane, i.e. at $t=x$, if the following boundary conditions are imposed on the physical fermions $\Theta_{3,11}$ 


$$
\left.\Theta_{3,11}^{\dot{\alpha} \dot{Y}}\right|_{t=x}=\vartheta^{\dot{\alpha}} \otimes \epsilon_{1}^{\dot{Y}},\left.\quad \Theta_{3,11}^{\dot{\alpha} Y}\right|_{t=x}=\psi^{\dot{\alpha}} \otimes \epsilon_{2}^{Y},
$$

where $\dot{Y}(Y)$ are chiral( anti-chiral) spinors indices on $\mathcal{X}$.

One can identify zero modes living on the boundary of the M2-brane $\vartheta^{\dot{\alpha}} \otimes \epsilon_{1}^{\dot{Y}}$ with the supersymmetry broken by the M2-brane. This is compatible with our considerations in section 3. Indeed, exactly these components of background supersymmetry are broken for the instanton described by the embedding eq.(52).

The bosonic part of the M2-action is

$$
S_{M 2}=Z, \quad Z=R \beta_{i} a^{i} x-i \hat{\mathcal{A}}, \quad \hat{\mathcal{A}}=\mathcal{A}\left(\beta_{i} a^{i}\right)-x\left(\beta_{i} \chi^{i}\right) .
$$

where, as in section two, $\beta_{i}\left[\sum_{2}^{i}\right]$ is the homology class of the boundary curve.

Now, by evaluating the embedding matrices for an M5-brane up to linear order in $\vartheta$ and solving the equation in the (6|16) superspace ( see [35])

$$
d B=H+f^{*} \mathbf{C},
$$

we obtain the expression for $B_{y \bar{y}}^{(2)}$ up to linear order in $\vartheta$

$$
B_{y \bar{y}}^{(2)}=A_{y \bar{y}}^{(2)}+i \partial_{y} X^{n} \partial_{\bar{y}} X^{\bar{m}}\left(-\vartheta \Gamma_{n \bar{m}} \bar{\psi}+C_{n \bar{m} \hat{P}} \vartheta \Gamma^{\hat{P}} \bar{\psi}\right)
$$

In solving equation (65) we have used constraints on the superform $H$. Specifically, we have used the condition that the only non-vanishing components of the superform $H$, in the basis $e^{A}$, are the components $H_{a b c}$ with all three bosonic indices. This was derived in [35] by requiring $\kappa$-supersymmetry of the action of an M2-brane ending on an M5-brane.

In (66) we have dropped terms containing derivatives of the fluctuating fields such as $\partial_{\mu} \mathcal{A}$, since these terms in the vertex operator will not contribute to the fermion two-point function we wish to compute.

Now, from eq.(50) and eq.(66), and using the properties (30) of covariantly constant spinors on $\mathcal{X}$, we evaluate the interaction between zero-modes of fermions living on an M2brane boundary $\vartheta_{\dot{\alpha}}$ and fermions $\psi_{\dot{\alpha}}$ to be

$$
V_{\psi}=i\left(\beta_{i} a^{i}\right) \vartheta^{\dot{\alpha}} \psi_{\dot{\alpha}}
$$


Note that the contribution to the interaction from the second boundary term in eq.(66) was cancelled by the term from the bulk, after integrating by parts, due to the presence of the piece in the embedding (52) which was quadratic in fermions.

The last step in deriving the "vertex operator" for the chiral fermion superpartner of $Z$, denoted $\chi_{\dot{\alpha}}$, is to relate $\psi_{\dot{\alpha}}$ and $\chi_{\dot{\alpha}}$. To achieve this we consider a supersymmetric variation of $Z$ with supersymmetry parameter $\left(\epsilon^{\dot{\alpha}} \otimes \epsilon_{1}, \epsilon^{\alpha} \otimes \epsilon_{2}\right)$. The result is

$$
\delta Z=\left(R \beta_{i} a^{i}\right) \delta x+x \delta\left(R \beta_{i} a^{i}\right)-i \delta \hat{\mathcal{A}}
$$

where

$$
\begin{gathered}
\delta x=i \epsilon \Gamma^{(11)} \bar{\psi}=-\frac{i}{R}\left(\epsilon^{\dot{\alpha}} \psi_{\dot{\alpha}}+\epsilon^{\alpha} \psi_{\alpha}\right) \\
\delta \hat{\mathcal{A}}=\left(\beta_{i} a^{i}\right)\left(\epsilon^{\dot{\alpha}} \psi_{\dot{\alpha}}-\epsilon^{\alpha} \psi_{\alpha}\right)
\end{gathered}
$$

Equation (70) is a direct consequence of eq.(66) and the definitions (23,64).

Denoting by $\lambda_{\dot{\alpha}}^{i}$ the superpartners of the bulk scalars $T^{i}$, we get the desired relation

$$
\chi_{\dot{\alpha}}=2\left(\beta_{i} a^{i}\right) \psi_{\dot{\alpha}}+x\left(\lambda_{\dot{\alpha}}^{i} \beta_{i}\right)
$$

and hence the "vertex operator" for $\chi_{\dot{\alpha}}$ is

$$
V_{\chi}=\frac{i}{2} \vartheta^{\dot{\alpha}} \chi_{\dot{\alpha}}
$$

\section{The case of one M5-brane.}

In this section we will discuss the scalar potential for the case of one M5-brane located at position $y=x$ on the M-theory interval. The general formula has been quoted above in (11). In order to evaluate this expression we need both $K$ and $W$. We will describe first $W$ and then $K$, and then put them together.

\section{$5.1 \quad$ Superpotential}

In the present setting the superpotential $W$ can be written as a sum of 5 pieces

$$
W=W_{\text {pert }}+W_{2}+W_{3}+W_{4}+W_{5}
$$

which have the following origins: 
- $W_{\text {pert }}$ is the Yukawa superpotential for the charged chiral superfields, given in ([47, 17])

$$
W_{\text {pert }}=\frac{(4 \pi) \sqrt{2}}{3} \lambda_{\hat{I}_{1} \hat{I}_{2} \hat{I}_{3}} C^{\hat{I}_{1}} C^{\hat{I}_{2}} C^{\hat{I}_{3}},
$$

The Yukawa couplings are given by

$$
\lambda_{\hat{I}_{1} \hat{I}_{2} \hat{I}_{3}}=\int \Omega \wedge u_{I_{1}}^{x_{1}} \wedge u_{I_{2}}^{x_{2}} \wedge u_{I_{3}}^{x_{3}} f_{x_{1} x_{2} x_{3}}^{\left(\mathcal{R}_{1} \mathcal{R}_{2} \mathcal{R}_{3}\right)}
$$

and depend on the complex structure and bundle moduli, but are independent of $T^{i}$ and $S . f_{x_{1} x_{2} x_{3}}^{\left(\mathcal{R}_{1} \mathcal{R}_{2} \mathcal{R}_{3}\right)}$ projects onto the singlet in $\mathcal{R}_{1} \times \mathcal{R}_{2} \times \mathcal{R}_{3}$ ( if it exists), and $\Omega$ is a choice of nowhere zero holomorphic $(3,0)$ form on $\mathcal{X}$.

- $W_{2}$ is the sum of two pieces coming from an M2-brane stretched between the M5-brane and the boundary 9-brane at $y=0$ or $y=1$ respectively. We have shown in the previous section that

$$
W_{2}=h\left\{\exp (-Z)+\exp \left(-\left(\beta_{i} T^{i}-Z\right)\right)\right\},
$$

(Note that the relative sign can be changed by a shift in the imaginary part of $T$ or Z.)

- $W_{3}$ is the contribution to the superpotential due to gaugino condensation studied in [15, 17, 国. It is given by

$$
W_{3} \sim \exp \left(-\frac{3}{2 b_{0}} S\right)
$$

where $b_{0}$ is a beta-function coefficient for the gauge group on the second 9-brane. We are working in a region of moduli space constrained by (11). It follows that

$$
3 V \gg 2 b_{0} R a^{i} \beta_{i} x, \quad 3 V \gg 2 b_{0} R a^{i} \beta_{i}(1-x)
$$

and hence the contribution of $W_{3}$ to the potential is much smaller than the contribution of $W_{2}$, and will henceforth be neglected.

- $W_{4}$ is the contribution from M2-brane instantons stretched between the two "M9branes." The contribution from a single membrane wrapping a holomorphic curve $\Sigma \subset \mathcal{X}$ has the form 


$$
W_{4} \sim \exp \left(-\beta_{i} T^{i}\right)
$$

In the case of the standard embedding ( with no 5-branes) the sum over all such curves in a fixed homology class vanishes. This happens because $W_{4}$ is just the world-sheet instanton contribution to the superpotential in the effective theory near a $(2,2)$ vacuum of the weakly coupled heterotic string. Such superpotentials for moduli are known to be zero (24, 25, 26]). $W_{4}$ is also zero for the special cases of the "non-standard embedding" arising in weakly coupled heterotic $(0,2)$ vacua which are related to linear sigma models. For example, $W_{4}=0$ for the quintic in $P^{4}$. Nevertheless, it is expected that these corrections will be nonzero for generic $(0,2)$ compactifications [13, 14].

- $W_{5}$ is the superpotential coming from an M5-brane wrapping the whole $\mathcal{X}$.

$$
W_{5} \sim \exp \left(-\tau_{M 5} S v\right)=\exp \left(-\frac{1}{4} S\right)
$$

$W_{5}$ is of the same order as $W_{3}$ and again we can neglect it relative to the effects of open membranes.

\subsection{Kahler potential for bulk moduli and charged scalars}

To evaluate the scalar potential in (1) one also needs the Kahler potential f

$$
K=K_{S}+K_{T}+K_{m}+K_{c p l x}+K_{5}+K_{\text {bundle }}
$$

The first four pieces in this expression have already been obtained in previous papers. We will derive a formula for $K_{5}$ below. It would be interesting to learn more about $K_{\text {bundle }}$, but we will not do so in this paper. In this section we review the results for the first four terms, obtained in [16, 17, 48, 49, 50].

The first two terms in (81) are:

$$
K_{S}=-\ln (S+\bar{S}), \quad K_{T}=-\ln \left(\frac{1}{6} d_{i j k}\left(T^{i}+\bar{T}^{i}\right)\left(T^{j}+\bar{T}^{j}\right)\left(T^{k}+\bar{T}^{k}\right)\right)
$$

\footnotetext{
${ }^{4}$ Here we are assuming that the moduli space is a product space, as is valid in our approximation.
} 
To leading order in an expansion in $C^{I}$ the charged matter has a Kähler potential of the form

$$
K_{m}=Z_{\hat{I} \hat{J}} C^{\hat{I}} \bar{C}^{\hat{J}}+\cdots
$$

Here $Z_{\hat{I} \hat{J}}$ is constructed from the metric for bundle moduli $G_{B \hat{I} \hat{J}}$ as follows. First of all, $G_{B}$ is defined by

$$
G_{B \hat{I} \hat{J}}=\frac{1}{v V} \int_{\mathcal{X}} \sqrt{g} g^{m \bar{m}} u_{I m x} u_{J \bar{m}}^{x}
$$

and depends on the Kahler moduli $a^{i}$, as well as on the complex structure and bundle moduli. Next we define $K_{B \hat{I} \hat{J}}:=e^{\frac{K_{T}}{3}} G_{B \hat{I} \hat{J}}$. Note that the dependence on the Kahler moduli is only through the combination $T^{i}+\bar{T}^{i}$. Then we can define

$$
Z_{\hat{I} \hat{J}}=G_{B \hat{I} \hat{J}}-\frac{e^{-\frac{K_{T}}{3}} 2 \xi_{i}}{S+\bar{S}} \tilde{\Gamma}_{B \hat{I} \hat{J}}^{i}
$$

where $\xi_{i}$ was defined in (16), and

$$
\begin{gathered}
\Gamma_{B \hat{I} \hat{J}}^{i}=K_{T}^{i j} \frac{\partial K_{B \hat{I} \hat{J}}}{\partial T^{j}}, \quad K_{T i j}=\frac{\partial^{2} K_{T}}{\partial T^{i} \partial \bar{T}^{j}} \\
\tilde{\Gamma}_{B \hat{I} \hat{J}}^{i}=\Gamma_{B \hat{I} \hat{J}}^{i}-\left(T^{i}+\bar{T}^{i}\right) K_{B \hat{I} \hat{J}}-\frac{2}{3}\left(T^{i}+\bar{T}^{i}\right)\left(T^{k}+\bar{T}^{k}\right) K_{T k j} \Gamma_{B \hat{I} \hat{J}}^{j},
\end{gathered}
$$

$K_{T}^{i j}$ denote the inverse of the matrix $K_{T i j}$.

In formulae (85) and (83) the following restrictions on the scalar fields are assumed

$$
\begin{gathered}
Z_{\hat{I} \hat{J}} C^{\hat{I}} \bar{C}^{\hat{J}} \ll 1 \\
\frac{2 \xi_{i}}{S+\bar{S}} \tilde{\Gamma}_{B \hat{I} \hat{J}}^{i} \ll K_{B \hat{I} \hat{J}}
\end{gathered}
$$

The Kahler function for the complex structure moduli is

$$
K_{c p l x}=-\ln \left(\overline{\Pi^{a}} \mathcal{G}_{a}\right), \quad \mathcal{G}_{a}=\partial_{\Pi^{a}} \mathcal{G}, \quad a=1, \ldots, h_{21}+1
$$

and can be expressed in terms of the periods over the A-cycles $\Pi^{a}$ and the prepotential $\mathcal{G}$, with complex structure moduli expressed as $\pi^{\alpha}=\frac{\Pi^{\alpha}}{\Pi^{0}}, \quad a=(0, \alpha)$. 


\subsection{Kahler potential for the M5-brane moduli}

The last piece in (81) is $K_{5}$, the Kahler potential giving the kinetic terms for the 5-brane scalars $x$ and $\mathcal{A}$. As we were finishing our project we found that ([41]) obtained $K_{5}$ in the special case of $h^{(1,1)}=1$. Since we got our result independently and in a different way, we will explain the derivation below.

To find $K_{5}$ we start from the bosonic part of the Pasti-Sorokin-Tonin action for the M5-brane [37]

$$
\begin{gathered}
S_{M 5}=\tau_{M 5} \int_{W_{6}} d^{6} y\left(-\sqrt{-\operatorname{det}\left(\gamma_{M N}+i H_{M N}\right)}-\frac{1}{4} \sqrt{-\gamma} v_{L} H^{* L M N} H_{M N P} v^{P}\right) \\
+\tau_{M 5} \int_{W_{6}}\left(\hat{C}_{6}+\frac{1}{2} d A_{2} \wedge \hat{C}_{3}\right)
\end{gathered}
$$

Here the tension of the M5-brane is $\tau_{M 5}=\frac{1}{(2 \pi)^{5}} M_{11}^{6}$. The other terms in the action are defined by

$$
\begin{gathered}
\gamma_{M N}=\frac{\partial X^{\hat{M}}}{\partial y^{M}} \frac{\partial X^{\hat{N}}}{\partial y^{N}} g_{\hat{M} \hat{N}}^{(11)}, \quad H^{M N}=H^{* M N P} v_{P}, \quad H^{* M N P}=-\frac{1}{3 ! \sqrt{-\gamma}} \epsilon^{M N P L K Q} H_{L K Q} \\
H_{M N P}=3 \partial_{[M} A_{N P]}^{(2)}-\left(\hat{C}_{3}\right)_{M N P}, \quad \hat{C}_{M N P}=\frac{\partial X^{\hat{M}}}{\partial y^{M}} \frac{\partial X^{\hat{N}}}{\partial y^{N}} \frac{\partial X^{\hat{P}}}{\partial y^{P}} C_{\hat{M} \hat{N} \hat{P}} \\
\hat{C}_{M_{1} \ldots M_{6}}=\frac{\partial X^{\hat{M}_{1}}}{\partial y^{M_{1}}} \ldots \frac{\partial X^{\hat{M}_{6}}}{\partial y^{M_{6}}} C_{\hat{M}_{1} \ldots \hat{M}_{6}} \quad v_{N}=\frac{\partial_{N} \Phi}{\sqrt{\partial_{K} \Phi \partial^{K} \Phi}}, \quad v_{N} v^{N}=1 .
\end{gathered}
$$

where $\Phi$ is the PST scalar and $\hat{C}_{6}$ is the magnetic dual of $\hat{C}_{3}$.

We wish to do Kaluza-Klein reduction of the above action along the holomorphic curve $\Sigma$. We split the coordinates in the bulk as $X^{\hat{M}}=\left(\xi^{\mu}, X^{a}, X^{\bar{a}}, X^{11}\right)$ where $a, \bar{a}=1, \ldots, 3$ are indices for the complex coordinates, and $\xi^{\mu}$ are coordinates along the noncompact $R^{4}$. We choose $\mu$ to run over $\mu=0,1,2,5$. . The coordinates along the worldvolume $W_{6}$ of the 5-brane are taken to be $y^{M}$ which we split as 4 real coordinates $y^{\mu}, \mu=0,1,2,5$ and one complex coordinate $y$ along the holomorphic curve $\Sigma$. A natural gauge choice for the PST scalar is [38]

$$
v_{M}=\delta_{M}^{5}, \quad A_{5 M}=0
$$

\footnotetext{
${ }^{5}$ We have changed notation from section two for this computation.
} 
While the gauge choice (92) breaks six dimensional covariance, after the KK reduction we will obtain a covariant 4-dimensional action.

The massless fluctuations of the M5 brane are described by fields

$$
X^{11}=\pi \rho x\left(\xi^{\mu}\right), \quad A_{m n}\left(\xi^{\mu}\right), \quad \mathcal{A}\left(\xi^{\mu}\right), \quad \mu=(m, 5), m=0,1,2
$$

where $\mathcal{A}\left(\xi^{\mu}\right)$ was defined in equation (23). Keeping only terms of quadratic order in derivatives we obtain the following 4-dimensional action!

$$
\begin{gathered}
S_{M 5}=-v^{\frac{1}{3}} \tau_{M 5} \int_{W_{4}}\left\{\frac{1}{2}(\pi \rho)^{2} \frac{e\left(a^{i} \beta_{i}\right) R}{V}\left(\partial_{\mu} x\right)\left(\partial^{\mu} x\right)-\frac{1}{2} \frac{\left(a^{i} \beta_{i}\right) R V}{e g^{55}}\left(H^{y \bar{y}}\right)^{2}\right. \\
+\frac{1}{2}(\pi \rho)^{2} \frac{e}{\left(a^{i} \beta_{i}\right) R V}\left[\partial_{m} \hat{\mathcal{A}}+x \partial_{m}\left(\beta_{i} \chi^{i}\right)\right] g_{(3)}^{m n}\left[\partial_{n} \hat{\mathcal{A}}+x \partial_{n}\left(\beta_{i} \chi^{i}\right)\right] \\
\left.+(\pi \rho) \frac{g^{5 m}}{g^{55}}\left[\partial_{m} \hat{\mathcal{A}}+x \partial_{m}\left(\beta_{i} \chi^{i}\right)\right] H^{y \bar{y}}+(\pi \rho)\left[\partial_{5} \hat{\mathcal{A}}+x \partial_{5}\left(\beta_{i} \chi^{i}\right)\right] H^{y \bar{y}}+(\pi \rho)^{2} \frac{e x}{V^{2}} \partial^{\mu} \sigma \partial_{\mu}\left[\mathcal{A}\left(\beta_{i} a^{i}\right)\right]\right\}
\end{gathered}
$$

where $e=\sqrt{-\operatorname{det} g_{\mu \nu}}$, while $g^{5 m}$ and $g^{55}$ are components of the inverse of the 4-dimensional metric $g^{\mu \nu}$. One should take care that $g_{(3)}^{m n}$ is the inverse of the 3 -dimensional metric so that

$$
g^{m n}=g_{(3)}^{m n}+\frac{g^{5 m} g^{5 n}}{g^{55}} .
$$

Moreover, in (93) we have

$$
H^{y \bar{y}}=\frac{1}{2} \epsilon^{m n p}\left[\partial_{m} A_{n p}-\partial_{m} X^{11} C_{11 n p}\right]
$$

(where we have used (91)) and finally we have also introduced

$$
\hat{\mathcal{A}}=\mathcal{A}\left(\beta_{i} a^{i}\right)-x\left(\beta_{i} \chi^{i}\right), \quad \partial^{m} \sigma=g^{m \nu} \partial_{\nu} \sigma
$$

One can see from (93) that integrating out $H^{y \bar{y}}$ gives

$$
H^{y \bar{y}}=(\pi \rho) \frac{e}{\left(\beta_{i} a^{i}\right) R V} g^{5 \mu}\left[\partial_{\mu} \hat{\mathcal{A}}+x \partial_{\mu}\left(\beta_{i} \chi^{i}\right)\right]
$$

Plugging the expression for $H^{y \bar{y}}$ back into (93) restores 4-dimensional covariance and results in the action

$$
\kappa_{4}^{2} S_{M 5}=-\int_{W_{4}} e\left\{\frac{1}{2} \frac{\left(a^{i} \beta_{i}\right) R}{V}\left(\partial_{\mu} x\right)\left(\partial^{\mu} x\right)+\frac{1}{2} \frac{1}{\left(a^{i} \beta_{i}\right) R V}\left[\partial_{\mu} \hat{\mathcal{A}}+x \partial_{\mu}\left(\beta_{i} \chi^{i}\right)\right]^{2}\right.
$$

${ }^{6}$ We are grateful to Steuard Jensen for pointing out a mistake in the original version of the equation below. 


$$
\left.+\frac{x}{V^{2}} \partial^{\mu} \sigma \partial_{\mu}\left[\mathcal{A}\left(\beta_{i} a^{i}\right)\right]\right\}
$$

One can now extract the Kahler potential for the 5-brane moduli. The terms in the action (94) uniquely determine $K_{5}$ to be

$$
K_{5}=\frac{(Z+\bar{Z})^{2}}{(S+\bar{S})\left(\beta_{i} T^{i}+\beta_{i} \bar{T}^{i}\right)},
$$

A check of the supergravity kinetic terms associated with $K_{5}$ shows that but there are extra terms coming from (95) and given by:

$$
\begin{gathered}
-\int_{W_{4}} e\left\{-x\left(a^{i} \beta_{i}\right) R V^{-2}\left(\partial_{\mu} x\right)\left(\partial^{\mu} V\right)-\frac{1}{2} x^{2} V^{-2}\left(\partial_{\mu}\left(R\left(a^{i} \beta_{i}\right)\right)\left(\partial^{\mu} V\right)\right.\right. \\
\left.-\frac{x^{2}}{2 V^{2}} \partial_{\mu}\left(\beta_{i} \chi^{i}\right)\left(\partial^{\mu} \sigma\right)-\frac{x\left(\beta_{i} \chi^{i}\right)}{V^{2}} \partial_{\mu} x \partial^{\mu} \sigma+\frac{1}{2} x^{2}\left(a^{i} \beta_{i}\right) R V^{-3}\left(\partial_{\mu} V \partial^{\mu} V+\partial_{\mu} \sigma \partial^{\mu} \sigma\right) \cdot\right\}
\end{gathered}
$$

These terms are exactly cancelled by the terms coming from $K_{S}=-\ln (S+\bar{S})$ after including an $x$-dependent correction to the definition of the chiral field $S$

$$
S=V+i \sigma+T^{i} \beta_{i} x^{2}
$$

Note, that the above correction $T^{i} \beta_{i} x^{2}$ is of order $\mathcal{E}^{e f f}$ with respect to $V$. There are no $x$-dependent corrections to the other fields at this order.

The proper interpretation of these facts is that the complex structure on field space is corrected at the nonlinear level by (97) and that the Kähler potential $K_{S}+K_{5}$ should be written as

$$
\widehat{K_{S}}=-\log \left[S+\bar{S}-\frac{(Z+\bar{Z})^{2}}{\left(\beta_{i} T^{i}+\beta_{i} \bar{T}^{i}\right)}\right]
$$

It would be interesting to learn if this expression is valid at higher order in the expansion in $Z$.

\subsection{Potential in the case $h^{(1,1)}=1$.}

In the previous sections we have given formulae valid for a generic $\mathcal{X}$. We will now specialize to the case of $h^{(1,1)}=1$ in order to simplify the analysis of the potential. As we have stressed above, in this case there is no net contribution to the non-perturbative potential from M2-branes stretched between the two boundary 9-branes, i.e. $W_{4}=0$. 
When $h^{(1,1)}=1$ the dependence on the Kahler parameter $a$ of the metric (84) for the bundle moduli can be easily extracted by a scaling argument. We can choose a basis $u_{I m}^{x}$ that does not depend on $a$. Then the inverse of Calabi-Yau metric scales like

$$
g_{C Y}^{m \bar{m}}=\frac{1}{a} \omega_{(1)}^{m \bar{m}}
$$

where $\omega_{(1), n \bar{m}}$ is, say, an integral generator of $H^{(1,1)}(\mathcal{X}) \cap H^{2}(\mathcal{X}, Z)$. Under these conditions the Kahler metric for charged scalars (86) simplifies considerably and is given by

$$
Z_{\hat{I} \hat{J}}=\left(\frac{3}{T+\bar{T}}+\frac{2 \xi}{S+\bar{S}}\right) H_{\hat{I} \hat{J}}, \quad \xi=\beta^{(0)}+\beta(1-x)^{2},
$$

where $H_{\hat{I} \hat{J}}$ depends only on complex structure and bundle moduli. In the case at hand $H_{2}(\mathcal{X}, Z)$ is of rank 1 and generated by a rational curve $\Sigma$. We take $\beta=1$, which corresponds to wrapping a 5-brane only once around $\Sigma$.

The perturbative potential for charged scalars was obtained in [17]. Using the formulae from the appendix we have calculated the non-perturbative potential including explicit leading dependence on Kahler moduli and charged scalars. As mentioned in the introduction we write the full potential in the form

$$
\left(\kappa_{4}\right)^{4} U=\left(U_{0}+U_{1}+U_{2}\right)
$$

where, as mentioned in the introduction, we organize terms by the order in the nonperturbative superpotential. $U_{0}$ begins with the perturbative potential. $U_{1}$ results from mixing between the perturbative and nonperturbative contributions to $W$, while $U_{2}$ is the term of second order in the nonperturbative superpotential. The formula for the potential contains a prefactor $e^{K}$. We have used the explicit results for $K_{S}$ and $K_{T}$, and we have dropped $K_{m}$ and $K_{5}$ since they contribute subleading effects to the order we are working.

We now give the leading expressions for the three terms in $(\sqrt[100]{)})$ in more detail. The leading contributions to $U_{0}$ are given by

$$
U_{0}=\frac{4 \pi^{2}}{3 \tilde{d}} \frac{e^{K_{\mathrm{cplx}}+K_{\text {bundle }}}}{V J^{2}}|\lambda C C|^{2}+U_{D}
$$

In the above formulae $V=\tilde{d} a^{3}, \quad J:=R a$, where $d=6 \tilde{d}$ is the intersection number on $\mathcal{X}$. The expression

$$
|\lambda C C|^{2}=C^{\hat{I}_{1}} C^{\hat{I}_{2}} \lambda_{\hat{I}_{1} \hat{I}_{2} \hat{I}_{3}} H^{\hat{I}_{3} \hat{J}_{3}} \bar{\lambda}_{\hat{J}_{1} \hat{J}_{2} \hat{J}_{3}} \bar{C}^{\hat{J}_{1}} \bar{C}^{\hat{J}_{2}}
$$


comes from derivatives of $W$ with respect to $C^{\hat{I}}$.

The $D$-term is given by

$$
U_{D}=\frac{18 \pi^{2}}{V J^{2}} \sum_{(a)}\left(\bar{C}^{\hat{I}} H_{\hat{I} \hat{J}} T^{(a)} C^{\hat{J}}\right)^{2}
$$

where $T^{(a)}$ are generators of the unbroken four dimensional gauge group $H$, and we are assuming there are no induced FI parameters.

To leading order $U_{1}$ is given by:

$$
U_{1}=-\frac{e^{K_{\text {cplx }}+K_{\text {bundle }}}(1-x)}{2 \tilde{d} V J^{2}}\left\{e^{-J x} \operatorname{Re}\left(W_{\text {pert }} \bar{h} e^{i \alpha_{1}}\right)-e^{-J(1-x)} \operatorname{Re}\left(W_{\text {pert }} \bar{h} e^{i \alpha_{2}}\right)\right\}
$$

where we define the axion fields

$$
\alpha_{1}=\operatorname{Im} Z, \quad \alpha_{2}=\operatorname{Im}(T-Z),
$$

There will be corrections from terms higher order in the expansion in $\frac{|C|^{2}}{J}, \mathcal{E}^{e f f}, \mathcal{E}_{R}^{e f f}$. There are also corrections from multiply-wrapped membranes.

The leading contribution to $U_{2}$ is a two-instanton term

$$
\begin{aligned}
U_{2}= & \frac{e^{K_{\text {cplx }}+K_{\text {bundle }}}}{8 \tilde{d} J^{2}}|h|^{2}\left\{e^{-2 J x}+e^{-2 J(1-x)}-2 e^{-J} \cos \left(\alpha_{1}-\alpha_{2}\right)\right. \\
& \left.+\frac{2 J}{3 V}(1-2 x) e^{-2 J(1-x)}+\frac{4 J x}{3 V} e^{-J} \cos \left(\alpha_{1}-\alpha_{2}\right)\right\}+\cdots
\end{aligned}
$$

The leading piece comes from $K^{Z \bar{Z}}\left|\partial_{Z} W\right|^{2}$. Note that in the second line of (106) we have kept terms which are formally higher order in our expression since they multiply $J / V \sim \mathcal{E}^{e f f}$. We have kept these because, near $x=1 / 2, \quad \cos \left(\alpha_{1}-\alpha_{2}\right)=1$ the leading piece vanishes. At these points the order $J / V$ corrections which come from $K^{Z \bar{Z}}$ and the prefactor $e^{K}$ multiply zero and we can legitimately say that the leading term near $x=1 / 2, \quad \cos \left(\alpha_{1}-\alpha_{2}\right)=1$ is given by the last term in the second line of (106). We will need these subleading terms in section 5.5 below. Of course, there are many other corrections of relative order $\mathcal{O}\left(\left(\mathcal{E}^{e f f}\right)^{p}\left(\mathcal{E}_{R}^{e f f}\right)^{q}, \frac{|C|^{2}}{J}\right)$, where $p \geq 1$ and $q>0$.

The region of validity of our result for the potential, (100), is constrained by several considerations.

- We must assume that all sizes are much bigger than the 11D Plank length. 


$$
\pi \rho R x \gg l_{11}, \quad \pi \rho R(1-x) \gg l_{11}, \quad a^{\frac{1}{2}} v^{\frac{1}{6}} \gg l_{11}
$$

and from these conditions it follows, in particular, that $J x \gg 1, \quad J(1-x) \gg 1$.

- Since we are working to quadratic order in the Kähler potential in a series expansion in $C$ we must have

$$
|C|^{2}:=C^{\hat{I}} H_{\hat{I} \hat{J}} \bar{C}^{\hat{J}} \ll J
$$

- The effective expansion parameters should be small, or, equivalently,

$$
V \gg J, \quad J^{2} \gg V
$$

- We must be able to drop $\mathcal{E}^{e f f}$ corrections to each of the 9 terms in the potential. We count all the terms wich have different sructure,i.e. 5 from $U_{2}, 2$ from $U_{1}$ and 2 from $U_{0}$.

$$
U=\sum_{a=1}^{9} u_{a}\left(1+f_{a} \mathcal{E}^{e f f}\right)
$$

iff

$$
\mathcal{E}^{e f f}\left|\sum_{a=1}^{9} f_{a} u_{a}\right| \ll\left|\sum_{a=1}^{9} u_{a}\right|
$$

Given our ignorance regarding $f_{a}$ we will assume that they are $O(1)$ and impose the stronger condition

$$
\mathcal{E}^{e f f} \sum_{a=1}^{9}\left|u_{a}\right| \ll\left|\sum_{a=1}^{9} u_{a}\right|
$$

- Finally, as mentioned in the introduction, we should stress that we are assuming that we are working at a generic smooth point in the complex structure and bundle moduli space.

\subsection{5-brane dynamics}

We can get some heuristic idea about the 5-brane dynamics by considering the theory on a finite volume of 3-dimensional space and keeping only the spatially homogeneous modes of the scalar fields. Even in this drastic approximation the resulting system is a very complicated 
dynamical system described by a particle mechanics Lagrangian with the (very) schematic form

$$
\operatorname{vol}\left(\text { space) } \int d t\left\{\left(\frac{\dot{V}}{V}\right)^{2}+\left(\frac{\dot{J}}{J}\right)^{2}+\frac{(\dot{C})^{2}}{J}+\frac{(\dot{J} x+\dot{x} J)^{2}}{V J}-U\right\}\right.
$$

where the potential energy is

$$
\begin{aligned}
& U=\frac{1}{V J^{2}}\left(\alpha C^{4}-\beta(1-x)|C|^{3}\left|e^{-J x} \mp e^{-J(1-x)}\right|+\right. \\
& \left.\quad+\gamma V\left[\left(e^{-J x} \mp e^{-J(1-x)}\right)^{2}+\frac{2 J}{3 V}(1-2 x) e^{-2 J(1-x)} \pm \frac{4 J x}{3 V} e^{-J}\right]\right)
\end{aligned}
$$

We have only kept the real parts of the fields. The philosophy behind this is that by a "Born-Oppenheimer" type approximation we expect that the axions will relax much more rapidly than the real parts into the most attractive channel. The choice of \pm depends on what term is dominating, $U_{1}$ or $U_{2}$.

The positive constants $\alpha, \beta$ and $\gamma$ are functions of the complex structure and bundle moduli, but these are being held fixed in this discussion.

While the dynamical system we must study is rather complicated we can get some heuristic idea of what to expect in three distinct regions within the region of validity of our potential.

- In one region the charged scalar fields are zero, while $J$ and $V$ are large. The leading contributions to the potential are positive and decrease with increasing $J, V$. In this region the 5 -brane leads to a repulsive interaction between the M9 walls. Setting $C=0$ and choosing "- " sign to set axions in the most attractive channel in eq.(112) we get:

$$
U \sim \gamma \frac{1}{J^{2}}\left\{\left(e^{-J x}-e^{-J(1-x)}\right)^{2}+\frac{2 J}{3 V}(1-2 x) e^{-2 J(1-x)}+\frac{4 J x}{3 V} e^{-J}\right\}
$$

Note that $U$ has a minimum in $x$ at $x=\frac{1}{2}$. Expanding around the minimum $x=\frac{1}{2}+y$, the resulting potential is

$$
U=\gamma\left\{\frac{2}{3 V J} e^{-J}+4 y^{2} e^{-J}\right\}
$$

Now we can see the need for keeping the last two terms in the expression for $U_{2}$ in eq. (106). Although we are neglecting the $\mathcal{E}^{e f f}$-corrections to the Kahler potential, such corrections multiply the leading three terms in eq.(106) which sum up to zero at $x=\frac{1}{2}$. On the other hand, the terms we have written, and which follow from the leading pieces 
in $K$ become the leading terms at the stationary points $x=\frac{1}{2}$. Consequently, $J$ flows towards infinity and $x$ moves towards the middle of the interval. We must assume $V^{1 / 2} \ll J \ll V$ to stay within the region of validity.

- If the charged scalar fields are important in such a way that

$$
\left|U_{1}\right| \gg U_{2}
$$

then the leading $x$-dependent term in the potential is attractive:

$$
U=\alpha C^{4}-\beta(1-x)|C|^{3}\left(e^{-J x}+e^{-J(1-x)}\right)
$$

Note that in this case we have to choose the "+" sign in eq.(112) if the axions are in the most attractive channel. Thus, if $x<1 / 2$ the 5 -brane moves towards the wall at $y=0$, and if $x>1 / 2$ the 5 -brane moves towards the wall at $y=1$. Note, that in each of these two subregions $U_{0} \gg\left|U_{1}\right|$ as a direct consequence of (115). Since $U_{0}$ is the dominant term $J$ and $V$ will evolve to large values. Since the $C$ field is simultaneously evolving a more careful analysis of the dynamical system would be highly desirable. But we will not do that here.

More generally, one can show that the potential (112) is non-negative at a generic point in the bundle and complex strucure moduli space, within in our region of validity (107-110), and thus predicts decompactification of both the Calabi-Yau and the orbifold interval. The argument, which is straightforward but long can be found in Appendix B.

Note that (112) is the leading potential only under our assumption that we work at a generic smooth point in bundle and complex structure moduli space. It would be interesting to incorporate singularities in complex structure and bundle moduli space in the discussion. There are potentially many new terms in the potential that must be reconsidered. It is possible that using the known results on complex structure and bundle moduli space one can address this problem.

\subsection{Conflicting instabilities}

One interesting consequence of the discussion in the previous section is that there is a strong coupling dual of the Dine-Seiberg problem where the M-theory interval (and the CalabiYau) tends to decompactify. In the case of heterotic M-theory with the standard embedding 
(i.e. no 5-branes) this has already been discussed by Banks and Dine [5], who noted that one can use holomorphy to extrapolate the weak coupling superpotential based on gluino condensation. In the presence of an M5-brane (in the case $h^{(1,1)}=1$ ) the above formulae show that in the region specified by (107 110) there is a similar effect due to open membrane instantons.

It is of some interest to compare the above result with what we expect for the weakly coupled heterotic string, since our considerations are only valid at large heterotic string coupling. Indeed, the heterotic coupling is related to the length of the M-theory interval by

$$
R \rho \sim l_{11}\left(g_{s}\right)^{\frac{2}{3}}
$$

and we require $\pi \rho R \gg l_{11}$. In the regime of weak coupling and large $V$, the potential has been discussed in [27]. It was shown there that the effective potential is positive and behaves as

$$
U_{e f f} \sim e^{-\frac{V}{g_{s}^{2}}}
$$

This favors an evolution to weak coupling $g_{s} \rightarrow 0$ and large volume $V \rightarrow \infty$. One might worry that the calculations of $([27)$ were performed in the case of the standard embedding, and in backgrounds with other $E_{8} \times E_{8}$ gauge instantons one must take into account the contribution of world-sheet instantons as well [13, 14]. Nevertheless, as we have repeatedly mentioned, these effects often sum to zero [21, 22] so once again and we can use (116) in the region of small $R$ and large $V$.

In view of the above, we can combine our result (100) with (116), to learn that the "true" potential goes to zero through positive values in both limits $R \rightarrow 0$ and $R \rightarrow \infty$.

This indicates that there must be a stationary point somewhere in the intermediate region, i.e. at some finite value of $R$. The nature of the stationary points that lie in the middle of moduli space is unknown, and is, of course, an interesting and outstanding question.

\section{Multiple covers and chirality changing transitions}

It is of considerable interest to determine the nature of the low energy theory in the limit that the M5 moves into the the boundary 10-manifold. In this section we will make some 
comments on this limit. We will need to make some guesses and the results of this section are not as rigorous as in the previous sections. For definiteness we will consider the limit $x \rightarrow 0$.

One good reason for studying the limit $x \rightarrow 0$ is that there are strong indications that in such limits there can be very interesting chirality-changing phase transitions in the low energy theory. This was discovered, in the present context, by Kachru and Silverstein [59]. The theory of these transitions has been considerably extended to many new examples in [44].

The main new ingredient that is needed to discuss the $x \rightarrow 0$ behavior is a multiple-cover formula for the open membrane instantons. The fact that there must be nontrivial effects from multiply-wrapped M2 branes (at least for those stretching between two 5-branes) can be seen by considering the holomorphy of the full superpotential $W$ as a function of $Z_{i}-Z_{j}$ [8]. The instanton effects must be suppressed by a factor proportional to the volume of the stretched membrane and therefore must behave like $\exp \left[\mp\left(Z_{i}-Z_{j}\right)\right]$ for $\pm \operatorname{Re}\left(Z_{i}-Z_{j}\right)>0$. This is only consistent with holomorphy if there is an infinite series with at best a finite radius of convergence.

Multiply-wrapped worldsheet instanton corrections to $d=4, N=2$ prepotentials are known to have a universal form $f(n, \Sigma)$ for an $n$-times wrapped curve $\Sigma$ where $f$ only depends on the topology of $\Sigma$ [60, 61, 65, 62, 63, 64]. Since worldsheet instantons are special cases of M2-brane instantons we will make the working hypothesis that there is similarly a multiple cover factor $f(n, \Sigma)$ for M2-brane instanton corrections to the superpotential $W$. Some evidence for this can be found in [57, 58, 56]. Unfortunately, the topologies studied in the above papers do not contain our case of $P^{1} \times[0,1]$. Therefore we will take

$$
\Delta W=h \sum_{n=1}^{\infty} f(n) e^{-n Z}+e^{i \delta} h \sum_{n=1}^{\infty} f(n) e^{-n(T-Z)}
$$

and make the rather weak assumption that the asymptotic behavior of $f(n)$ for large $n$ is $f(n) \sim n^{m} e^{J x_{0} n}$ for some constants $m$ and $x_{0}$. For simplicity we set $x_{0}=0$ although there could in principle be a shift in the location of the small instanton transition.

The constants $m$ and $\delta$ above are unknown, but we can make some comments on them. First, the relative phase $e^{i \delta}$ was not important in the 1-instanton sector, where we can change the relative phase by shifting the axion $\operatorname{ImT}$. It does become a nontrivial issue in the multi- 
instanton sectors. Nevertheless, for our analysis of the dynamics in the subregion $R e Z \ll 1$ the second piece in (117) is negligible, so the issue need not concern us here.

Next, let us consider the power $m$ in the asymptotic behavior of $f(n)$. If we wish the chiral fermion mass term in standard supergravity to be a single-valued function of $Z$ in a region surrounding $Z=0$, then $m$ cannot be a negative integer such that $|m|>2$. Singlevaluedness implies that the monodromy of $\partial_{Z} \partial_{Z} W$ around $Z=0$ should be diagonalizable thus excluding a singularity of the type $Z^{n} \log Z, n \geq 2$ in $W$. I

Let us now re-consider the region of validity of our expression. The infinite series for $\Delta W$ can be obtained reliably in the region $\operatorname{Re} Z \gg 1$ (where we can use 11-dimensional supergravity) and then analytically continued to the region where $\operatorname{Re} Z=J x \ll 1$. To ensure that corrections to the Kahler function are small one must still require that

$$
V \gg 1, \quad V^{\frac{1}{2}} \ll J \ll V, \quad|C|^{2} \ll J
$$

Since these conditions do not imply $J x \gg 1$ or $J(1-x) \gg 1$ we can study the physics of the 5-brane approaching the boundary.

For definiteness and simplicity let us now assume that $f(n)=n^{m}$ for some constant $m$. Then we have

$$
\partial_{Z} \Delta W=-h L i_{-(m+1)}\left(e^{-Z}\right)
$$

where $L i$ is a polylogarithm function

$$
L i_{-(m+1)}(t)=\sum_{n=1}^{\infty} n^{m+1} t^{n}
$$

In this case the leading order contribution to $U_{1}$ is given by:

$$
U_{1}=-\frac{e^{K_{\mathrm{cplx}}+K_{\text {bundle }}(1-x)}}{2 \tilde{d} V J^{2}} \operatorname{Re}\left[\bar{W}_{\text {pert }} h L i_{-(m+1)}\left(e^{-Z}\right)\right]
$$

while the leading contribution to $U_{2}$ is

$$
U_{2}=\frac{e^{K_{\mathrm{cplx}}+K_{\mathrm{bundle}}}}{8 \tilde{d} J^{2}}|h|^{2}\left|L i_{-(m+1)}\left(e^{-Z}\right)\right|^{2}
$$

\footnotetext{
${ }^{7}$ There are known examples of logarithmic superpotentials for $n=0,1$ that make good physical sense. This is usually related to some kind of pair creation phenomena. We thank K. Hori for very useful discussions on this issue.
} 
In all the cases below we will assume that the axion phases are in the maximally attractive channel.

One interesting limit is $Z \rightarrow 0$. Here we can use the behaviour of the polylogarithm

$$
L i_{-(m+1)}\left(e^{-Z}\right) \sim Z^{-(m+2)}, \quad Z \rightarrow 0
$$

(for $m=-2$ we replace $Z^{0}$ by $\log Z$ ) to write out (schematically ) the leading potential at $J x \ll 1$

$$
\begin{gathered}
U=\frac{1}{V J^{2}}\left(\alpha C^{4}-\beta(1-x) \frac{|C|^{3}}{(J x)^{2+m}}+\gamma \frac{V}{(J x)^{4+2 m}}\right), \quad m>-2 \\
U=\frac{1}{V J^{2}}\left(\alpha C^{4}+\beta|C|^{3}(1-x) \log (J x)+\gamma V(\log J x)^{2}\right), \quad m=-2
\end{gathered}
$$

where $\alpha, \beta, \gamma$ are positive functions of complex structure and bundle moduli as above and we have set $\operatorname{Im} Z=0$.

Therefore, for small enough $J x$ (holding the other moduli fixed) the leading term in the potential (121), (122) is

$$
\frac{\gamma}{J^{2}(J x)^{4+2 m}}, \quad m>-2, \quad \frac{\gamma(\log J x)^{2}}{J^{2}}, \quad m=-2
$$

and there is a repulsive force on the 5-brane. Indeed there is an infinite energy barrier forbidding the 5 brane from hitting the wall.

One should not conclude from the above that there will be no chirality changing transition, since the axionic degree of freedom in $Z$ can change the qualitative features of the potential drastically. Unfortunately, in order to study this question in detail just knowing the asymptotic behavior will not suffice and one needs a precise version of the formula for $f(n)$ in order to work out the analytic continuation from $\operatorname{Re}(Z) \gg 1$ to $\left|e^{-Z}\right|=1$. For definiteness we will consider $f(n)=n^{m}$ where $m \geq-2$ is integral.

Let us consider first the case $m \geq 0$. We take $Z=J x+i \pi$, where $J x \ll 1$ and expand $e^{-Z}=-1+J x-\frac{1}{2}(J x)^{2}$. We use the Taylor expansion for the polylogarithm

$$
L i_{-(m+1)}(-1+t)=L i_{-(m+1)}(-1)-L i_{-(m+2)}(-1) t+\frac{1}{2}\left(L i_{-(m+3)}(-1)-L i_{-(m+2)}(-1)\right) t^{2}
$$

and the following useful relations

$$
L i_{-m}(-1)=\left(2^{1+m}-1\right) \zeta(-m), \quad \zeta(-2 k)=0, \quad \zeta(1-2 k)=-\frac{B_{2 k}}{2 k}, \quad k=1,2, \ldots
$$


where $\zeta$ is the Riemann $\zeta$-function and $B_{k}$ are Bernoulli numbers taken in the convention:

$$
\frac{y}{e^{y}-1}=1-\frac{1}{2} y+\frac{B_{2}}{2 !} y^{2}+\frac{B_{4}}{4 !} y^{4}+\ldots
$$

Substituting $t=J x-\frac{1}{2}(J x)^{2}$ in the above Taylor expansion and keeping only terms up to $(J x)^{2}$ we have

$$
\begin{gathered}
L i_{-(m+1)}(-1+t)=(-1)^{k+1}\left(\nu_{1}^{k}-\nu_{2}^{k}(J x)^{2}\right), \quad m=2 k, \quad k=0, \ldots \\
L i_{-(m+1)}(-1+t)=(-1)^{k+1} 2 \nu_{2}^{k} J x, \quad m=2 k+1, \quad k=0, \ldots
\end{gathered}
$$

where we define positive numbers $\nu_{1}^{k}, \nu_{2}^{k}$

$$
\nu_{1}^{k}=\left(2^{2 k+2}-1\right) \frac{\left|B_{2 k+2}\right|}{2(1+k)}, \quad \nu_{2}^{k}=\left(2^{2 k+4}-1\right) \frac{\left|B_{2 k+4}\right|}{4(2+k)} .
$$

We now analyze the potential separately for the cases of even and odd $m$. For even $m=2 k$ we have the following leading potential at $J x \ll 1$

$$
U=\frac{1}{V J^{2}}\left(\alpha C^{4}-\beta(1-x)|C|^{3}\left(\nu_{1}^{k}-\nu_{2}^{k}(J x)^{2}\right)+\gamma V\left(\left(\nu_{1}^{k}\right)^{2}-2 \nu_{1}^{k} \nu_{2}^{k}(J x)^{2}\right)\right)
$$

If $C \neq 0$ then for sufficiently small $x$ the potential is attractive. If $C=0$ the potential is repulsive.

Now, for odd $m=2 k+1$ the leading potential is

$$
U=\frac{1}{V J^{2}}\left(\alpha C^{4}-2 \beta \nu_{2}^{k}(1-x) J x|C|^{3}+\gamma V\left(2 \nu_{2}^{k}\right)^{2}(J x)^{2}\right)
$$

Now the situation is opposite to the previous case. For $C \neq 0$ there is a repulsive force and if $C=0$ an attractive force.

Finally we analyze what happens for the cases $m=-1$ and $m=-2$, assuming $\operatorname{Im} Z=\pi$ and $J x \ll 1$. If $m=-1$ the leading potential is

$$
U=\frac{1}{V J^{2}}\left(\alpha C^{4}-\frac{1}{2} \beta|C|^{3}(1-x)\left(1-\frac{1}{2} J x\right)+\frac{\gamma V}{4}(1-J x)\right)
$$

The force on the 5-brane is attractive only if one allows for a large vev for $|C|^{3}$

$$
\beta|C|^{3}>\gamma V
$$


This is in principle possible since we only assume that $|C|^{3} \ll V^{\frac{3}{2}}$ and for large $V$ both inequalities can be satisfied. If $m=-2$ the leading potential is

$$
U=\frac{1}{V J^{2}}\left(\alpha C^{4}-\beta|C|^{3}(1-x)\left(\log 2-\frac{1}{2} J x\right)+\gamma V \log 2(\log 2-J x)\right)
$$

and the force is attractive only if

$$
\beta|C|^{3}>2(\log 2) \gamma V
$$

In both cases $m=-1$ and $m=-2$, attraction is only possible for large vev of charged scalars.

The general conclusion based on the analysis of various cases is that the physics of what happens when the 5-brane approaches the wall depends strongly on the detailed form of the multiple-cover formula.

Finally, let us comment on the relevance of this compututation to the examples studied by Ovrut, Pantev, and Park in [44]. One might at first conclude that in these examples the superpotential must vanish since the five-brane wraps a high genus curve. However, the curve wrapped by the 5 -brane is not irreducible and not isolated. It can very well happen that in the long-distance expansion of the M5 and M2 Lagrangians there are terms with many fermions (typically multiplying factors involving curvature tensors) which can lift the many fermion zeromodes associated with the nonisolated high genus curve. Thus, the question of whether or not a superpotential is generated is a complicated and difficult one, involving a discussion of the measure on the moduli space of the curve and the integral over that moduli

space. Considerations based on the global form of the moduli space for these curves based on the results of [66] do not appear to exclude the generation of such superpotentials.

\section{The case of $\mathrm{N}$ M5-branes.}

We will now briefly consider the potential in the case that there are N M5-branes at positions $x_{1}<x_{2}<\ldots<x_{N}$. We will assume for simplicity that all the 5-branes are wrapped over the same rational curve $\Sigma$, so that open M2-instantons can be stretched between any pair of 5-branes. Moreover, to simplify the analysis we assume that the 5-branes are more 
or less evenly separated. Finally, we restrict our consideration only to the leading nonperturbative potential, so we do not take into account 2-instanton contributions to $W$ and we need only consider M2-branes between neighboring 5-branes. Similarly, we only keep the contribution of 5-9 instantons coming from M2-instantons stretching between the boundary and the nearest M5-brane. Under these conditions we will have

$$
R\left(x_{n}-x_{n-1}\right) \pi \rho \gg l_{11}, \quad \forall n=1, \ldots, N+1 .
$$

Neglecting $\mathcal{E}^{e f f}$ corrections due to the distortion of the background, the Kähler function for the collection of 5-branes will be just a sum of Kahler functions for each 5-brane.

The potential is again given by formula (100), with the same conditions on the region of validity. The 2 -instanton terms in the potential $U_{2}$, which dominate at $C=0$, are:

$$
U_{2}=\frac{e^{K_{\text {cplx }}+K_{\text {bundle }}}}{8 \tilde{d} J^{2}}|h|^{2} \sum_{n=1}^{N}\left\{e^{-2 J\left(x_{n+1}-x_{n}\right)}+e^{-2 J\left(x_{n}-x_{n-1}\right)}-2 e^{-J\left(x_{n+1}-x_{n-1}\right)} \cos \left(\tilde{\alpha}_{n}\right)\right\}+\cdots
$$

where we denote

$$
\tilde{\alpha}_{n}=\left\{a\left(2 \mathcal{A}_{n}-\mathcal{A}_{n+1}-\mathcal{A}_{n-1}\right)+\chi\left(x_{n+1}+x_{n-1}-2 x_{n}\right)\right\}, n=1, \ldots, N
$$

and $x_{0}=0, x_{N+1}=1, \mathcal{A}_{0}=\mathcal{A}_{N+1}=0$. If instead we assume that

$$
x_{1} \gg x_{n}-x_{n-1}, \quad\left(1-x_{N}\right) \gg x_{n}-x_{n-1}, \quad \forall 1<n \leq N
$$

and choose a special subregion where $\cos \tilde{\alpha}_{n}=0, \forall n$, then the potential has the form of a non-periodic Toda-chain potential. (The kinetic energies are the standard ones, in our approximation.) As is well known, Toda theory has an exact solution, where all particles move away from each other [43]. In heterotic M-theory this signals an instability in the time evolution of the positions of M5-branes along the orbifold interval: they tend to run away from each other. At the same time $R a$ evolves to infinity. In short, the system explodes.

Using again a "Born-Oppenheimer" type approximation we expect that the axions will relax much more rapidly than the real parts into the most attractive channel $\cos \tilde{\alpha}_{n}=1, \quad \forall n$. This implies that the evolution with a Toda-like potential is unstable because of the axions.

When the charged vevs are nonzero we should consider instead the term $U_{1}$ in the potential arising from cross terms between perturbative and nonperturbative pieces. This is given 
by

$$
\begin{gathered}
U_{1}=-\frac{e^{K_{\text {cplx }}+K_{\text {bundle }}}|h| \zeta}{2 \tilde{d} J^{2} V}\left\{e^{-J x_{1}}\left(1-x_{1}\right) \cos \left(\tilde{\gamma}_{1}\right)-e^{-J\left(1-x_{N}\right)}\left(1-x_{N}\right) \cos \left(\tilde{\gamma}_{N}\right)\right. \\
\left.-\sum_{n=1}^{N-1}\left(x_{n+1}-x_{n}\right) e^{-J\left(x_{n+1}-x_{n}\right)} \cos \left(\tilde{\phi}_{n}\right)\right\}
\end{gathered}
$$

where $W_{\text {pert }}=\zeta e^{i \phi_{1}}, h=|h| e^{i \phi_{h}}$ are decompositions into modulus and phase and

$$
\begin{gathered}
\tilde{\gamma}_{1}=\operatorname{Im} Z_{1}+\phi_{1}-\phi_{h}, \quad \tilde{\gamma}_{N}=\operatorname{Im}\left(T-Z_{N}\right)+\phi_{1}-\phi_{h}, \\
\tilde{\phi}_{n}=\operatorname{Im}\left(Z_{n+1}-Z_{n}\right)+\phi_{1}-\phi_{h}, \quad n=1, \ldots, N-1
\end{gathered}
$$

\section{Possible future directions and applications}

A central question in heterotic M-theory is the existence of isolated minima of the potential for moduli. While most of our results predict runaway or unstable behavior (as expected) we have seen some encouraging hints. We have argued that the potential must have nontrivial stationary points in moduli space. We have also seen that a good place to look for interesting behavior of the potential is at singular loci in complex structure and bundle moduli space. For example, if one allows some of the coefficients $\alpha, \beta, \gamma$ in sections 5.5 and section 6 to vanish it is easy to imagine scenarios where the potential predicts compactification, rather than decompactification.

There are many technical issues raised by the above computations which should be solved and which moreover can be solved with presently available technology.

One circle of questions includes finding the appropriate generalization of the multiplecover formula for worldsheet instantons. A related set of questions concerns effects associated with membranes wrapping higher genus curves and nonisolated curves in $\mathcal{X}$. As we have seen in section six, results on these questions would have very interesting physical applications.

A second circle of questions concerns the possibility of obtaining a more concrete understanding of the dependence of the membrane determinants as functions of the complex structure moduli. It might be possible to find classes of compactifications in which one can 
give fairly explicit formulae for the dependence on gauge bundle moduli, although this might prove to be challenging.

Beyond the extensions mentioned above, which we believe are within reach, there loom far more difficult questions. One of the most challenging issues is to give a proper definition of Horava-Witten theory in a regime outside the validity of the expansion in $\left(\kappa_{11}\right)^{2 / 3}$. Another difficult, and pressing, problem is that of finding ways to make definite and quantitative statements about the Kähler potential of the effective supergravity theory in a wider range of validity.

Nevertheless, even given the limitations of our computations, the results do have some interesting ranges of validity. It might be quite interesting to study more thoroughly the dynamics, both classical and quantum mechanical of the moduli in the problem. In this paper we have limited ourselves to some very heuristic and naive pictures of the dynamics. It might also be interesting to see if there are any distinctive features of the "modular cosmology" resulting from the above potential for moduli [54].

\section{Acknowledgements}

We would like to thank Jeff Harvey and Marcos Mariño for collaboration at some stages of this research. We would also like to thank B. Acharya, T. Banks, D.-E. Diaconescu,

M. Douglas, S. Kachru, O.Ganor, S. Gukov, M. Mariño, A.Mikhailov, N.Nekrasov, and M.Rangamani for discussions and useful correspondence. The work of GM is supported by DOE grant DE-FG02-96ER40949. The work of GP is supported by the Rutgers DOE grant DE-FG02-96ER40949 and by the Yale DOE grant DE-FG02-92ER-40704.

\section{Appendix A}

For the convenience of the reader we list here the leading expressions for Kahler potential in the case $h^{(1,1)}=1$, together with formulae for the Kahler metric and inverse metric. The Kahler potential is:

$$
K=K_{S}+K_{T}+K_{m}+K_{c p l x}+K_{5}+K_{\text {bundle }}
$$




$$
\begin{gathered}
K_{S}=-\ln (S+\bar{S}), \quad K_{T}=-\ln \left(\tilde{d}(T+\bar{T})^{3}\right) \\
K_{5}=\frac{(Z+\bar{Z})^{2}}{(S+\bar{S})\left(\beta_{i} T^{i}+\beta_{i} \bar{T}^{i}\right)} \\
K_{m}=\left(\frac{3}{T+\bar{T}}+\frac{2 \xi}{S+\bar{S}}\right) H_{\hat{I} \hat{J}} C^{\hat{I}} \bar{C}^{\hat{J}}
\end{gathered}
$$

We now give the components of the Kahler metric on the space of scalars which have been used in section 5.4. We keep only leading terms in each of the component, neglecting corrections of the relative order $O\left(\mathcal{E}^{e f f}, \mathcal{E}_{R}^{e f f}, \frac{|C|^{2}}{R a}\right)$.

$$
\begin{gathered}
K_{S \bar{S}}=\frac{1}{4 V^{2}}, \quad K_{S \bar{T}}=\frac{x^{2}}{4 V^{2}}, \quad K_{S \hat{J}}=-\frac{\xi H_{\hat{I} \hat{J}} C^{\hat{I}}}{2 V^{2}}, \quad K_{S \bar{\alpha}}=-\frac{\xi \partial_{\bar{\alpha}} H_{\hat{\hat{I}} \bar{J}} C^{\hat{I} \bar{C}^{\bar{J}}}}{2 V^{2}} \\
K_{S \bar{Z}}=-\frac{x}{2 V^{2}}, \quad K_{T \bar{T}}=\frac{3}{(2 R a)^{2}}, \quad K_{\bar{T} I}=-\frac{3 H_{\hat{I} \hat{J}} \bar{C}^{\hat{J}}}{(2 R a)^{2}} \\
K_{\bar{T} \alpha}=-\frac{3 C^{\hat{I}} \partial_{\alpha} H_{\hat{I} \hat{J}} \bar{C}^{\hat{J}}}{(2 R a)^{2}} \quad K_{Z \bar{T}}=-\frac{x}{2 V R a}, \quad K_{\hat{I} \hat{J}}=\frac{3}{2 R a} H_{\hat{I} \hat{J}}, \quad K_{\hat{I} \bar{\alpha}}=\frac{3 \partial_{\bar{\alpha}} H_{\hat{I} \hat{J}} \bar{C}^{\hat{J}}}{2 R a} \\
K_{Z \hat{I}}=-\frac{(1-x) H_{\hat{I} \hat{J}} C^{\hat{J}}}{V R a}, \quad K_{\bar{Z} \alpha}=-\frac{(1-x) C^{\hat{I}} \partial_{\alpha} H_{\hat{I} \hat{J}} \bar{C}^{\hat{J}}}{V R a} \\
K_{\alpha \bar{\beta}}=K_{\alpha \bar{\beta}}^{(c p l x)}, \quad K_{Z \bar{Z}}=\frac{1}{2 V R a}
\end{gathered}
$$

Now, we solve the matrix equation

$$
K K^{-1}=1+O\left(\mathcal{E}^{\text {eff }}, \mathcal{E}_{R}^{\text {eff }}, \frac{|C|^{2}}{R a}\right)
$$

The inverse metric solving this equation is

$$
\begin{gathered}
K^{S \bar{S}}=4 V^{2}, \quad K^{T \bar{T}}=\frac{(2 R a)^{2}}{3}, \quad K^{\bar{T} \hat{J}}=C^{\hat{J}} \frac{2 R a}{3} \\
K^{\hat{I} \hat{J}}=\frac{2 R a}{3} H^{\hat{I} \hat{J}}, \quad K^{\bar{\alpha} \beta}=K_{c p l x}^{\bar{\alpha} \beta}, \quad K^{\hat{J} \beta}=-\partial_{\bar{\delta}} H_{\hat{I} \hat{K}} \bar{C}^{\hat{K}} H^{\hat{I} \hat{J}} K^{\bar{\delta} \beta} \\
K^{Z \bar{Z}}=2 R a V, \quad K^{Z \bar{S}}=4 R a V x, \quad K^{Z \bar{T}}=\frac{(2 R a)^{2}}{3} x, \quad K^{\hat{J} \bar{Z}}=\frac{(2 R a)}{3} C^{\hat{J}}(2-x)
\end{gathered}
$$

where the components not listed above are zero in our approximation. 


\section{Appendix B}

In section 5.5 we asserted that, within the region of validity of our computations, the potential is always positive. Here we give the detailed proof of that claim.

The only potentially negative term in the potential is $U_{1}$. We will show that it cannot be larger in magnitude than both of $U_{0}$ and $U_{2}$ in our region of validity.

First, imposing

$$
\left|U_{1}\right| \geq U_{2}
$$

means

$$
\beta(1-x)|C|^{3}\left(e^{-J x}+e^{-J(1-x)}\right) \geq \gamma V\left(e^{-J x}+e^{-J(1-x)}\right)^{2}
$$

It follows immediately that

$$
|C| \geq\left(\frac{\gamma V}{\beta}\left[e^{-J x}+e^{-J(1-x)}\right]\right)^{\frac{1}{3}}
$$

Now, at a generic point in bundle and complex moduli space, we have

$$
\alpha C^{4} \geq \alpha|C|^{3}\left(\frac{\gamma V}{\beta}\left[e^{-J x}+e^{-J(1-x)}\right]\right)^{\frac{1}{3}} \gg \beta|C|^{3}(1-x)\left(e^{-J x}+e^{-J(1-x)}\right)
$$

and we see that $U_{0} \gg\left|U_{1}\right|$.

Let us now assume

$$
\left|U_{1}\right| \geq U_{0}
$$

From this it follows that

$$
\beta(1-x)\left(e^{-J x}+e^{-J(1-x)}\right) \geq \alpha|C|
$$

and hence

$$
\left|U_{1}\right| \leq \frac{1}{J^{2} V} \frac{\beta^{4}}{\alpha^{3}}(1-x)^{4}\left(e^{-J x}+e^{-J(1-x)}\right)^{4}
$$

Let us consider, first, the region far enough from $x=1 / 2$. Then, for $x<\frac{1}{2}$, we have

$$
\left|U_{1}\right| \leq \frac{1}{J^{2} V} \frac{\beta^{4}}{\alpha^{3}}(1-x)^{4} e^{-4 J x}
$$

and

$$
U_{2} \sim \frac{1}{J^{2}} e^{-2 J x}
$$


As a consequence, $U_{2} \gg\left|U_{1}\right|$.

In the region close to $x=\frac{1}{2}$ we have instead, for sign "+" in eq.(137)

$$
\left|U_{1}\right| \leq \frac{1}{J^{2} V} \frac{\beta^{4}}{\alpha^{3}} e^{-2 J}
$$

and

$$
U_{2} \sim \frac{1}{J^{2}} e^{-J}
$$

and it follows immediately that

$$
U_{2} \gg\left|U_{1}\right|
$$

For sign "-" in eq.(137) the last statement is obvious.

\section{References}

[1] P. Hořava, E. Witten, "Heterotic and Type I String Dynamics from Eleven Dimensions" Nucl.Phys. B460 (1996) 506-524, hep-th/9510209

[2] P. Hořava, E. Witten, "Eleven-Dimensional Supergravity on a Manifold with Boundary", Nucl.Phys. B475 (1996) 94-114,hep-th/9603142

[3] E.Witten, "Strong Coupling Expansion Of Calabi-Yau Compactification", Nucl.Phys. B471 (1996) 135-158,hep-th/9602070

[4] T.Banks and M. Dine, "Phenomenology of Strongly Coupled Heterotic String Theory", Nucl.Phys.B479(1996)173.hep-th/9609046

[5] T.Banks and M. Dine, "Couplings and Scales in Strongly Coupled Heterotic String Theory", (hep-th/9605136)

[6] E.Witten, "Non-Perturbative Superpotentials In String Theory", Nucl.Phys. B474 (1996) 343-360 hep-th/9604030

[7] K.Becker, M.Becker, A.Strominger, Five-branes, Membranes and Non-perturbative String Theory. Nucl.Phys.B456:130-152,1995,hep-th/9507158

[8] J. Harvey, G.Moore, Superpotentials and Membrane Instanons, hep-th/9907026

[9] B. de Wit, K.Peeters, J. Plefka, "Open and Closed Supermembranes with Winding," Nucl.Phys.Proc.Suppl. 68 (1998) 206-215 
[10] E. Witten,World-Sheet Corrections Via D-Instantons, hep-th/9907041

[11] S. Kachru, S. Katz, A. Lawrence, J. McGreevy,Open string instantons and superpotentials Phys.Rev. D62 (2000) 026001; hep-th/9912151

[12] B.S. Acharya, M theory, Joyce Orbifolds and Super Yang-Mills,hep-th/9812205

[13] M.Dine, N.Seiberg, X.G.Wen, E.Witten, "Nonperturbative effects on the string worldsheet", Nucl.Phys. B278(1986)769-789

[14] M.Dine, N.Seiberg, X.G.Wen, E.Witten, "Nonperturbative effects on the string worldsheet (II)", Nucl.Phys. B289(1987)319-363

[15] P. Hořava , " Gluino Condensation in Strongly Coupled Heterotic String Theory", Phys.Rev. D54 (1996) 7561-7569,hep-th/9608019

[16] A. Lukas, B. A. Ovrut, D. Waldram, "Non-standard embedding and five-branes in heterotic M-Theory", Phys.Rev. D59 (1999) 106005, hep-th/9808101

[17] A. Lukas, B. A. Ovrut, D. Waldram, "Five-branes and Supersymmetry Breaking in M-theory", JHEP 9904 (1999) 009;hep-th/9901017

[18] A. Lukas, B. A. Ovrut, D. Waldram,K.Stelle, " Heterotic M-Theory in Five Dimensions, hep-th/9806051', Nucl.Phys. B552 (1999) 246-290

[19] R. Donagi, A. Lukas, B. A. Ovrut, D. Waldram " Non-Perturbative Vacua and Particle Physics in M-Theory", JHEP 9905 (1999) 018, hep-th/9811168

[20] R. Donagi, A. Lukas, B. A. Ovrut, D. Waldram, Holomorphic Vector Bundles and Non-Perturbative Vacua in M-Theory" JHEP 9906 (1999) 034,hep-th/9901009

[21] E.Silverstein, E.Witten, "Criteria for Conformal Invariance of $(0,2)$ Models", Nucl.Phys. B444 (1995) 161-190,hep-th/9503212

[22] J.Distler, S.Kachru,"Singlet Couplings and (0,2) Models", Nucl. Phys. B430 (1994) 13, hep-th/9406090

[23] P. Berglund, P. Candelas, X. de la Ossa, E. Derrick, J. Distler, T. Hubsch, On the Instanton Contributions to the Masses and Couplings of $E_{6}$ Singlets,Nucl. Phys. B454 (1995) 127;hep-th/9505164

[24] P. Candelas, X. de la Ossa, Moduli Space of Calabi-Yau Manifolds. Nucl.Phys.B355:455481,1991 
[25] A.Strominger," Special Geometry", Comm.Math.Phys.1333(1990)163.

[26] E.Witten, "Phases of N=2 Theories in two Dimensions", Nucl.Phys. B403(1993)159.hepth/9301042

[27] M.Dine, N.Seiberg, " Is the superstring weakly coupled?", Phys.Lett.B162(1985)299302; M.Dine, R.Rohm, N.Seiberg, E. Witten, Phys.Lett.B156(1985)55; M.Dine, N.Seiberg, Phys.Rev. Lett.55(1985)366

[28] P.Townsend, Brane Surgery Nucl. Phys. B., Proc. Suppl. 58 (1997) 163-175. hep-th 9609217

[29] A. Strominger Open P-Branes, Phys.Lett.B383:44-47,1996; ;hep-th/9512059

[30] P.S. Howe, E.Sezgin, Superbranes, Phys.Lett. B390 (1997) 133-142:hep-th/9607227; $\mathrm{D}=11, \mathrm{p}=5$, Phys.Lett. B394 (1997) 62-66; hep-th/9611008; P.S. Howe, E. Sezgin, P.C. West, Covariant Field Equations of the M Theory Five-Brane, Phys.Lett. B399 (1997) 49-59;hep-th/9702008

[31] D.Sorokin,Superbranes and Superembeddings, Phys.Rept. 329 (2000) 1-101 (hepth/9906142)

[32] E. Cremmer and S. Ferrara, Phys. Lett. 91B (1980) 61;

L. Brink and P. S. Howe, Phys. Lett. 91B (1980) 384.

[33] B. de Wit, K. Peeters, and J. Plefka, Superspace geometry for supermembrane backgrounds," Nucl.Phys. B532 (1998) 99;

[34] Y. Shibusa, "11-dimensional curved backgrounds for supermembrane in superspace,"

[35] E. Sezgin, P. Sundell," Aspects of the M5-Brane", hep-th/9902171

[36] M.Fabinger, P.Hořava," Casimir effect between world-branes in heterotic M-theory, hepth/0002073

[37] P. Pasti, D. Sorokin and M. Tonin, Physics Lett.B3989741, (hep-th/9701037);

I. Bandos, K. Lechner, A. Nurmagambetov, P. Pasti, D. Sorokin and M. Tonin, Phys. Rev. Lett.78974332, (hep-th/9701149).

[38] M. Aganagic, J. Park, C. Popescu, J. Schwarz, "World-Volume Action of the M Theory Five-Brane", Nucl.Phys. B496 (1997) 191-214:hep-th/9701166 
[39] J.Wess and J. Bagger,Supersymmetry and Supergravity,Second ed.,Princeton University Press 1992.

[40] E. Cremmer, S. Ferrara, L. Girardello, A. Van Proeyen, " Yang-Milles theories with local supersymmetry: Lagrangian, transformation laws and superhiggs effect," Nucl.Phys.B212:413,1983

[41] J.P.Derendinger, R.Sauser, "A five-brane Modulus in the Effective N=1 Supergravity of M-theory", hep-th/0009054

[42] Ph.Brax, J.Mourad, " Open Supermembranes Coupled to M-Theory Five-Branes", Phys.Lett. B416 (1998) 295-302; hep-th/9707246

[43] Perelomov.A, Integrable systems of classical mechanics and Lie algebras. Birkhuser Verlag, 1990.

[44] B.Ovrut, T.Pantev, J.Park: Small Instanton Transitions in Heterotic M-Theory, JHEP 0005 (2000) 045; hep-th/0001133

[45] C. Munoz, "Effective Supergravity from Heterotic M-Theory and its Phenomenological Implications," hep-th/9906152; D.G. Cerdeno and C. Munoz, "Phenomenology of NonStandard Embedding and Five-branes in M-Theory," hep-ph/9904444

[46] E. Bergshoeff, E. Sezgin, and P.K. Townsend, "Supermembranes and 11-dimensional supergravity," Phys. Lett. 189B(1987)75; "Properties of the Eleven-Dimensional Supermembrane Theory," Ann. Phys. 185(1988)330.

[47] M. Green, J.H. Schwarz, and E. Witten, Superstring Theory, Cambridge University Press, Cambridge (1987)

[48] T.Li, J.L.Lopez and D.V.Nanopoulos, Phys.Rev.D56,2602,(1997)

[49] E.Dudas,C.Grojean, Nucl.Phys.B507,553 (1997)

[50] H.P.Nilles, M.Olechowski, M.Yamaguchi, Phys.Lett.B415,24 (1997); Nucl.Phys.B530,43 (1998)

[51] S. Stieberger," (0,2) Heterotic Gauge Couplings and their M-Theory Origin", Nucl.Phys. B541 (1999) 109-144

[52] K. Choi, H. B. Kim, H. Kim, " Moduli Stabilization in Heterotic M-theory", Mod.Phys.Lett. A14 (1999) 125-134, hep-th/9808122 
[53] K. Benakli, "Phenomenology of Low Quantum Gravity Scale Models," Phys.Rev. D60 (1999) 104002

[54] T. Banks, M. Berkooz, G. Moore, S.H. Shenker, and P. Steinhardt, "Modular Cosmology," hep-th/9503114; Phys.Rev. D52 (1995) 3548-3562

[55] M. Atiyah, J. Maldacena, C. Vafa, "An M-theory Flop as a Large N Duality," hepth/0011256

[56] C. Vafa,"Superstrings and Topological Strings at Large N", hep-th/0008142

[57] H. Ooguri, C. Vafa, " Knot Invariants and Topological Strings",Nucl.Phys. B577 (2000) $419-438$

[58] S. Kachru, S. Katz, A. Lawrence, J. McGreevy, "Mirror symmetry for open strings", Phys.Rev. D62 (2000) 126005, hep-th/0006047

[59] S.Kachru, E.Silverstein, "Chirality Changing Phase Transitions in 4d String Vacua", Nucl.Phys. B504 (1997) 272-284, hep-th/9704185

[60] P.Candelas, X. C. de la Ossa. P.Green, L.Parkes," A pair of Calabi-Yau Manifolds as an exactly soluble superconformal theory", Essays on Mirror Manifolds, ed. S.T.Yau

[61] P.S. Aspinwall and D.R. Morrison, "Topological field theory and rational curves," Commun. Math. Phys. 151(1993)245

[62] M. Marino, G. Moore, "Counting higher genus curves in a Calabi-Yau manifold", Nucl.Phys. B543 (1999) 592-614, hep-th/9808131

[63] C. Faber and R. Pandharipande, "Hodge Integrals and Gromov-Witten Theory," math.AG/9810173

[64] R. Gopakumar, C. Vafa, " M-Theory and Topological Strings-I", hep-th/9809187; "MTheory and Topological Strings-II", hep-th/9812127

[65] D. R. Morrison, M. R. Plesser, "Summing the Instantons: Quantum Cohomology and Mirror Symmetry in Toric Varieties", Nucl. Phys. B440 (1995) 279-354, hep-th/9412236

[66] R. Donagi, B.A. Ovrut, and D. Waldram, "Moduli spaces of fivebranes on elliptic CalabiYau threefolds," hep-th/9904054 